Collection SFN 8 (2007) 179-205

(C) EDP Sciences, Les Ulis

DOI: $10.1051 / \mathrm{sfn}: 2007014$

\title{
Diffusion de neutrons aux petits angles appliquée aux études d'interfaces et de systèmes confinés
}

\author{
L. Auvray ${ }^{1}$ et A. Brûlet ${ }^{2}$ \\ ${ }^{1}$ LRP (UMR-7581), 2-8 rue Henri Dunant, 94320 Thiais, France \\ ${ }^{2}$ LLB (UMR12 CEA-CNRS), C.E. Saclay, 91191 Gif-sur-Yvette Cedex, France
}

\begin{abstract}
Résumé. La diffusion de neutrons aux petits angles (DNPA) permet de sonder la matière aux échelles spatiales allant de 0.5 et $50 \mathrm{~nm}$ en déterminant les grandeurs moyennes qui caractérisent la taille et la forme des objets ainsi que leurs interactions. Elle s'applique à divers systèmes, polymères, colloïdes, pores dans les solides ou amas dans les alliages...Elle permet d'étudier l'organisation en volume de ces systèmes, mais aussi en couches minces, sur des couches adsorbées ou greffées et même dans des milieux confinés. Ce cours expose les différentes grandeurs mesurables et les méthodes à utiliser pour y accéder. En particulier seront abordées les notions de facteur de forme et de facteur de structure et les différentes façons de jouer avec le contraste pour déterminer les structures d'objets complexes et leurs interactions. Ces notions seront d'abord appliquées à des exemples classiques d'études de systèmes poreux et de fondus de polymères. Ensuite, plusieurs exemples illustreront l'application de la diffusion de neutrons aux études de polymères aux interfaces ou confinés.
\end{abstract}

\section{INTRODUCTION}

La diffusion de neutrons aux petits angles (DNPA) sonde la structure moyenne («statique ») de la matière aux échelles spatiales allant de 0.5 et $50 \mathrm{~nm}$. Cette technique est utilisée pour déterminer les grandeurs moyennes qui caractérisent la taille et la forme des objets ainsi que leurs interactions. Elle s'applique à divers systèmes, des polymères, des colloïdes, des pores dans les solides ou des amas dans les alliages...Elle permet d'étudier l'organisation en volume de ces systèmes, mais aussi en couches minces, sur des couches adsorbées ou greffées et même dans des milieux confinés. Un atout majeur de la DNPA est la variation de contraste qui sera décrite plus loin ; elle est très utilisée, notamment en physico-chimie ou il est possible de remplacer les atomes d'hydrogène par leur isotope, le deutérium. Cette substitution isotopique permet de « voir » des objets dans leur milieu sans trop perturber leurs propriétés physiques.

L'expérience de DNPA consiste à envoyer un faisceau de neutrons de faible divergence incidente, de longueur d'onde moyenne $\lambda$, sur l'échantillon à étudier et à mesurer grâce à un détecteur la variation d'intensité diffusée $I(\theta)$ en fonction de l'angle de diffusion $\theta$.

En diffusion de neutrons aux petits angles, on considère qu'il n'y a pas d'échange d'énergie entre l'échantillon et les neutrons. Le paramètre physique est en fait le vecteur de diffusion dont le module est

$$
q=\frac{4 \pi}{\lambda} \sin \left(\frac{\theta}{2}\right)
$$

et sa dimension, l'inverse d'une longueur.

L'analyse de la courbe de diffusion $I(q)$ n'est possible qu'à deux conditions :

1. le domaine de q doit être du même ordre de grandeur que celui de l'inverse des distances à mesurer. Sachant que la longueur d'onde des neutrons est comprise entre 0.2 et $2 \mathrm{~nm}$, les valeurs de q intéressantes correspondant à des tailles caractéristiques comprises entre 0.5 et $50 \mathrm{~nm}$ ne sont accessibles qu'avec des angles de diffusion $\theta$ inférieurs à $10^{\circ}$.

2. l'intensité diffusée doit être mesurable, ce qui nécessite l'existence d'un contraste entre l'objet étudié et le milieu qui l'entoure. 
Ce problème de contraste a été évoqué à plusieurs reprises dans les cours précédents, notamment dans l'introduction de J. Teixeira (ce livre). Il a été détaillé dans plusieurs éditions d'écoles thématiques organisées par la Société Française de la Neutronique ; « Diffusion de Neutrons aux Petits Angles »[1] et « Neutrons et Magnétisme »[2]. Rappelons seulement qu'en diffusion de neutrons, le contraste peut être d'origine nucléaire ou magnétique. Dans ce chapitre, ne sera traitée que la diffusion nucléaire. Quelle qu'en soit l'origine, l'analyse des courbes de DNPA vise à déterminer notamment la taille globale des objets, i.e. le rayon de giration, leur forme et les interactions lorsque leur concentration dans l'échantillon augmente.

Ici, la description de la diffusion de neutrons sera limitée au strict nécessaire pour comprendre les résultats des expériences de DNPA. Le cours expose les différentes grandeurs mesurables et les méthodes à utiliser pour $y$ accéder. En particulier seront abordées : 1) les mesures effectuées aux petits vecteurs de diffusion qui sont liées aux fluctuations de concentration ; 2) les notions de facteur de forme et de facteur de structure ; 3) les différentes façons de jouer avec le contraste pour déterminer les structures d'objets complexes et leurs interactions. Ces notions seront d'abord appliquées à des exemples classiques d'études de systèmes poreux et de fondus de polymères. Ensuite, plusieurs exemples illustreront l'application de la diffusion de neutrons aux études de polymères aux interfaces ou confinés.

\section{SECTION EFFICACE DE DIFFUSION}

Le neutron est une particule neutre qui, contrairement aux rayons $\mathrm{X}$ ou à la lumière, interagit avec les noyaux en ignorant le cortège électronique. Son spin lui permet également d'interagir avec l'induction magnétique, et donc plus particulièrement les moments magnétiques des atomes de l'échantillon. Ce cas particulier de la diffusion par des systèmes magnétiques [3] ne sera pas traité dans ce chapitre.

\subsection{Diffusion par une assemblée d'atomes}

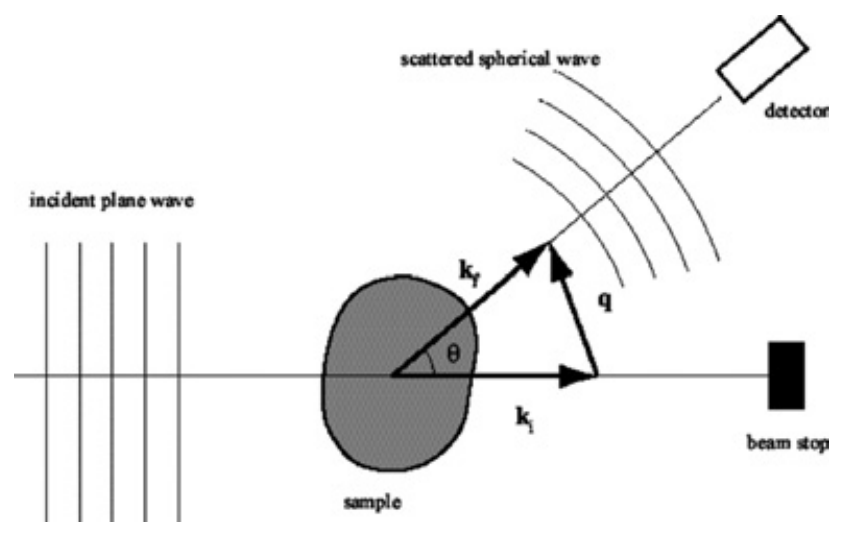

Figure 1. Schéma de principe de diffusion d'un rayonnement.

Lorsque l'onde plane associée au neutron, monochromatique de longueur d'onde $\lambda$ de vecteur d'onde incident $\vec{k}_{i}$, interagit avec un atome, elle est diffusée dans toutes les directions (voir Figure 1).

Si l'atome est à une position $\vec{r}$ par rapport à l'origine, l'onde diffusée dans la direction du vecteur d'onde $\vec{k}_{f}$, va être déphasée de $\vec{q} \cdot \vec{r}$, où $\vec{q}$ est le vecteur de diffusion relié à $\theta$ l'angle de diffusion :

$$
\vec{q}=\vec{k}_{f}-\vec{k}_{i} \quad|\vec{q}|=q=\frac{4 \pi}{\lambda} \sin \left(\frac{\theta}{2}\right)
$$


La probabilité qu'un neutron soit diffusé est caractérisée par une longueur de diffusion $\boldsymbol{b}$, dont le Tableau 1 reporte quelques valeurs. Les valeurs numériques de la longueur de diffusion, pour chaque élément de la classification périodique et de ses isotopes, sont déterminées expérimentalement [4] car les théories ne permettent pas de les calculer. La précision relative est bien souvent de l'ordre de $1 \%$. Elle peut être négative, et varie non seulement d'un atome à un autre, mais aussi d'un isotope à un autre, comme c'est le cas pour l'hydrogène $\left(b_{H}=-0.37410^{-12} \mathrm{~cm}\right)$ et pour le deutérium $\left(b_{D}=+0.667\right.$ $\left.10^{-12} \mathrm{~cm}\right)$. Ce dernier exemple est d'une grande importance en DNPA du fait que les longueurs de diffusion des deux isotopes sont de signes opposés et que par conséquent, celles des petites molécules élémentaires deutériées ou non deutériées pourront l'être également. Nous reviendrons sur cette propriété par la suite.

Table 1. Exemples de valeurs de longueurs de diffusion pour les neutrons et pour les rayons $\mathrm{X}$ à $q=0$. Issus de la référence [5].

\begin{tabular}{|c|c|c|c|l|}
\hline \multirow{2}{*}{$\begin{array}{c}\text { Numéro } \\
\text { atomique } Z\end{array}$} & $\begin{array}{c}\text { Eléments naturels } \\
\text { ou isotopes }\end{array}$ & $\begin{array}{c}\text { Longueur } \\
\text { de diffusion } \\
\text { cohérente } b \\
10^{-12} \mathrm{~cm}\end{array}$ & $\begin{array}{c}\text { Longueur } \\
\text { de diffusion } \\
\text { in cohérente } \\
10^{-12} \mathrm{~cm}\end{array}$ & $\begin{array}{l}\text { Longueur } \\
\text { de diffusion } \\
\text { cohérente } \\
10^{-12} \mathrm{~cm}\end{array}$ \\
\hline 1 & & -0.374 & 2.53 & 0.282 \\
& & +0.667 & 0.404 & 0.282 \\
\hline 6 & $\mathrm{H}$ & +0.665 & 0.005 & 1.69 \\
& $\mathrm{D}$ & +0.619 & 0.05 & 1.69 \\
\hline 28 & ${ }^{13} \mathrm{C}$ & 1.03 & 0.64 & \\
& $\mathrm{Ni}$ & 1.44 & 0 & 7.90 \\
& ${ }^{58} \mathrm{Ni}$ & 0.28 & 0 & \\
\hline
\end{tabular}

L'onde diffusée est sphérique. L'onde diffractée par une assemblée de $n$ noyaux est la somme des ondes sphériques diffractées par chaque noyau $i$, corrigées par un facteur de phase dû à la propagation de l'onde incidente pour arriver en $\vec{r}_{i}$. Les interférences produites (voir Figure 2) se calculent en fonction du vecteur de diffusion. On obtient alors dans l'approximation de Born et après développement au premier ordre pour $r$ grands [6] :

$$
\left.\varphi(\vec{r})=\exp \left(\overrightarrow{i k} \cdot r_{i}\right)-\frac{\exp \left(\overrightarrow{i k}_{f} \cdot \vec{r}\right)}{r} \sum_{i}^{n} b_{i} \cdot \exp \left(-i\left(\vec{k}_{f}-\vec{k}_{i}\right) \cdot \vec{r}\right)\right)
$$

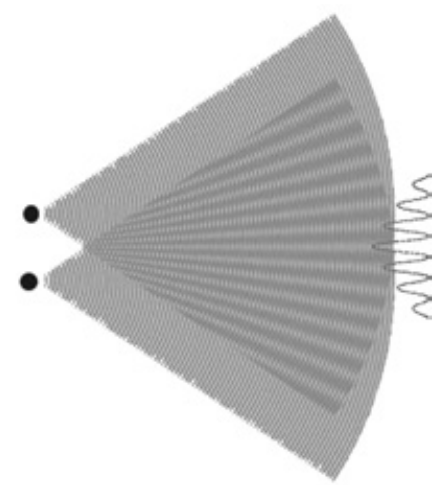

Figure 2. Interférences entre deux ondes sphériques diffusées par deux atomes distants de $\mathrm{r}$ dans l'espace réel. Le spectre d'interférences dépend de la distance entre les atomes et donc de la structure de l'échantillon. 
Le détecteur de neutrons n'étant pas sensible à la phase de l'onde, ne mesure pas l'amplitude de l'onde diffusée mais le carré de l'amplitude de l'onde. On définit la section efficace différentielle de diffusion $\Sigma(q)$ comme le nombre de neutrons comptés dans un angle solide $d \Omega$ du détecteur, et par unité de flux incident. Elle s'écrit simplement :

$$
\Sigma(q)=\sum_{i, j} b_{i} b_{j} \exp \left(-i \vec{q} \cdot\left(\vec{r}_{i}-\vec{r}_{j}\right)\right)
$$

où $i$ et $j$ sont des diffuseurs élémentaires de l'objet.

L'intensité diffusée est ainsi une mesure de la probabilité de trouver un atome de longueur de diffusion $b_{i}$ et un autre atome de longueur de diffusion $b_{j}$ séparés d'un vecteur $\vec{r}_{i}-\vec{r}_{j}$.

D'un point de vue théorique, l'amplitude de diffusion $A(\vec{q})$ est la transformée de Fourier de la densité $\rho(\vec{r})$ du système de $n$ atomes dans l'échantillon, pondérée par la longueur de diffusion $b_{i}$ de l'atome situé en $\vec{r}_{i} . \rho(\vec{r})=\sum_{i=1}^{n} \rho_{i}(\vec{r})=\sum_{i=1}^{n} \delta\left(\vec{r}-\vec{r}_{i}\right)$ où $\delta$ est la fonction de Dirac, $A(\vec{q})$ s'écrit : $A(\vec{q})=$ $\int_{V} e^{i q \cdot r} \cdot d_{3} \vec{r} \cdot \sum_{i} b_{i} \cdot \delta\left(\vec{r}-\vec{r}_{i}\right)$

Pour un ensemble de n noyaux du même isotope, sans spin, $\left(b_{i}=b\right)$, la section efficace de diffusion s'écrit de plusieurs façons :

$$
\Sigma(q)=\langle A(\vec{q}) A(-\vec{q})\rangle=\sum_{i, j}^{n, n} b^{2}\left\langle\exp \left(i \vec{q}\left(\vec{r}_{i}-\vec{r}_{j}\right)\right)\right\rangle=b^{2}\langle\rho(\vec{q}) \rho(-\vec{q})\rangle=b^{2} \int d_{3} \vec{r} \cdot e^{i \vec{q} \vec{r}} \cdot p(\vec{r})
$$

où les crochets désignent une moyenne d'ensemble sur les positions des noyaux. La fonction $p(\vec{r})$ est la fonction de corrélation de paire, c'est à dire la probabilité d'avoir deux atomes à une distance $r$.

Pour un ensemble de n noyaux du même élément ayant plusieurs isotopes, il faut tenir compte de la probabilité aléatoire qu'un site $r_{i}$ soit occupé par un isotope dans un état spin donné. Il faut faire des moyennes sur les longueurs de diffusion correspondantes. On peut montrer qu'alors la section efficace de diffusion est la somme de deux termes :

$$
\Sigma(\vec{q})=n \cdot b_{i n c}^{2}+b^{2}\langle\rho(\vec{q}) \rho(-\vec{q})\rangle ; \quad b_{i n c}^{2}=\left\langle b_{i}^{2}\right\rangle-\left\langle b_{i}\right\rangle
$$

Le premier terme de cette équation, appelé section efficace de diffusion incohérente, provient de l'absence de corrélation entre la position et l'état de spin ou isotopique du noyau. En DNPA, cette diffusion incohérente est indépendante de $q$. Elle est considérée comme un bruit de fond plat qu'il faudra soustraire [7,8]. Le second terme de l'équation (4) rend compte des fluctuations de densité de longueur de diffusion du système. C'est cette fonction, appelée section efficace de diffusion cohérente, que l'on cherche à mesurer. $b=\left\langle b_{i}\right\rangle$ est la longueur de diffusion cohérente.

Pour un ensemble d'éléments différents, chaque élément $\alpha$ comportant $n_{\alpha}$ atomes $\left(b_{\alpha}, b_{\mathrm{inc}}^{\alpha}\right)$, les équations (3) et (4) peuvent être généralisées :

$$
\Sigma(\vec{q})=\sum_{\alpha} n_{\alpha} \cdot b_{i n c}^{\alpha}+\sum_{\alpha, \beta}\left\langle b_{\alpha}\right\rangle\left\langle b_{\beta}\right\rangle \sum_{i, j}^{n_{\alpha}, n_{\beta}}\left\langle\exp \left(i \vec{q}\left(\vec{r}_{i}^{\alpha}-\vec{r}_{j}^{\beta}\right)\right)\right\rangle
$$

Le deuxième terme de cette équation est souvent écrit :

$$
\Sigma(\vec{q})=\sum_{\alpha, \beta} b_{\alpha} \cdot b_{\beta} S_{\alpha \beta}(\vec{q})
$$

où les fonctions de diffusion $S_{\alpha \beta}(\vec{q})$ sont les facteurs de structure partiels des différents éléments de l'ensemble. Ces fonctions ne dépendent que de la structure de l'échantillon et pas des contrastes.

L'équation générale de la diffusion (5) se simplifie en introduisant la notion de contraste dans la limite où l'échantillon est considéré comme incompressible. 


\subsection{Résolution spatiale}

En faisant l'approximation que les amplitudes diffusées par les deux éléments $i$ et $j$ n'interfèrent constructivement que si la distance $\left|\vec{r}_{i}-\vec{r}_{j}\right|$ est plus petite que $q^{-1}$, on a approximativement

$$
\exp \left(i \vec{q} \cdot\left(\vec{r}_{i}-\vec{r}_{j}\right)\right) \approx 0 \quad \text { si }\left|\vec{r}_{i}-\vec{r}_{j}\right|>q^{-1}
$$

et

$$
\exp \left(i \vec{q} \cdot\left(\vec{r}_{i}-\vec{r}_{j}\right)\right) \approx 1 \quad \text { si }\left|\vec{r}_{i}-\vec{r}_{j}\right|<q^{-1}
$$

Ces équations résument de manière simple deux règles essentielles de la diffusion :

(i) quand on observe l'intensité diffusée autour d'un certain angle et pour un certain vecteur de diffusion $q$, on observe la structure à une échelle spatiale et avec une résolution d'ordre $q^{-1}$,

(ii) à une échelle inférieure à $q^{-1}$, les amplitudes des éléments diffusants s'ajoutent, à une échelle supérieure à $q^{-1}$, les intensités des éléments diffusants s'ajoutent.

Pratiquement, elles traduisent le principe que dans un domaine de vecteur de diffusion $q$ donné, on ne verra que les fluctuations de longueur de diffusion sur des échelles de distances de l'ordre de $q^{-1}$. Ce principe (relié à des propriétés élémentaires de la transformation de Fourier) est une des clés pour comprendre la diffusion aux petits angles. Le module de vecteur de diffusion est simplement une loupe (voir la Figure 3) qui permet de déterminer la structure d'un objet à l'échelle $q^{-1}$, échelle que l'on peut faire varier aisément en DNPA sur deux à trois ordres de grandeurs.
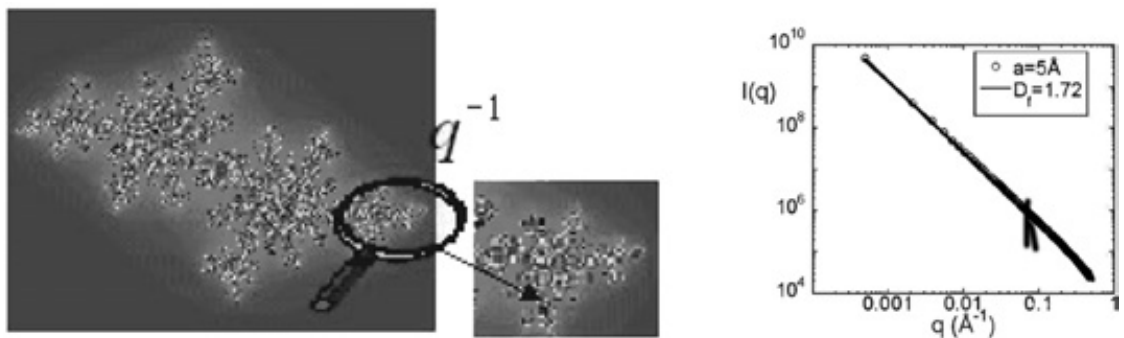

Figure 3. Observation à diverses échelles $q^{-1}$ de la structure auto-similaire d'un objet fractal. La diffusion de neutrons aux petits angles par une solution diluée de tels objets donne la dimension fractale de l'objet, ici $D_{f}=1.72$, sur une gamme de vecteurs de diffusion allant de l'inverse de sa dimension globale jusqu'à l'inverse de la taille élémentaire de l'objet a.

\section{INTENSITÉ DIFFUSÉE EN DNPA}

\subsection{Notion de diffuseur élémentaire. Compressibilité isotherme. Contraste}

Une des spécificités de la DNPA est la capacité d'explorer la structure de la matière à une échelle de distances, supérieures à $0.5 \mathrm{~nm}$. A cette échelle, on peut considérer que les diffuseurs élémentaires (DE) ne sont plus les noyaux, mais des molécules de tailles plus grandes. La longueur de diffusion cohérente de ces DE est la somme des longueurs de diffusion cohérente des atomes qui les composent. On trouve alors des valeurs très variables, voire même négatives comme les longueurs de diffusion des atomes seuls. Par exemple, la longueur de diffusion de l'eau légère vaut $b_{\mathrm{H}_{2} \mathrm{O}}=-0.16810^{-12} \mathrm{~cm}$ alors que celle de l'eau lourde est $b_{\mathrm{H}_{2} \mathrm{O}}=+1.9210^{-12} \mathrm{~cm}$.

Une deuxième conséquence de l'échelle de $q$ considérée provient de la très faible contribution des fluctuations de densité dans cette gamme de $q$. Si on considère un solvant simple, la thermodynamique 
[9] permet de montrer que l'extrapolation à $q$ égal 0 des fluctuations de densité de ce liquide, $\Sigma(0)=$ $b^{2}\left(\left\langle n^{2}\right\rangle-\langle n\rangle^{2}\right)$, est proportionnelle à sa compressibilité $\chi_{T}$ :

$$
\langle n\rangle \cdot k T \cdot \chi_{T}=v \cdot\left(\left\langle n^{2}\right\rangle-\langle n\rangle^{2}\right)
$$

où $k$ est la constante de Boltzmann et $T$ la température. Cette limite thermodynamique $\Sigma(0)$ est atteinte pour des vecteurs de diffusion $q^{*}$ de l'ordre de $6 \mathrm{~nm}^{-1}$. A des vecteurs de diffusion plus petits, $q<q^{*}$, ces fluctuations de densité sont faibles et souvent négligeables devant les fluctuations de concentration qui apparaissent lorsqu'on mélange deux solvants par exemple. Dans ces conditions $\left(q<q^{*}\right)$, on dit que les systèmes sont incompressibles.

Considérons maintenant un mélange de $\alpha=1,2, \cdots, m$ diffuseurs élémentaires, de longueur de diffusion $b_{\alpha}$, dans un volume $V$. Selon l'équation (5), la section efficace de diffusion cohérente de ce mélange, supposé isotrope, s'écrit :

$$
\Sigma(q)=\sum_{\alpha, \beta} b_{\alpha} \cdot b_{\beta} S_{\alpha \beta}(q)
$$

où les fonctions de diffusion $S_{\alpha \beta}(q)$ sont les facteurs de structure partiels des DE qui ne sont fonction que de $q$. Soit $\left\langle n_{\alpha}\right\rangle$ le nombre moyen de ces diffuseurs. Chacun d'eux occupe un volume élémentaire $v_{\alpha}=\frac{V}{\left\langle n_{\alpha}\right\rangle}$. Les relations (8) se généralisent en $[10,11]$ :

$$
\left\langle n_{\alpha}\right\rangle \cdot k T \cdot \chi_{T}=\sum_{\beta} v_{\beta} \cdot S_{\alpha \beta}(0)=\sum_{\beta} v_{\beta} \cdot S_{\alpha \beta}(q) \quad q<q^{*}
$$

En combinant les équations (9) et (10), il est alors possible d'éliminer un des DE, par exemple $\alpha=1$. On obtient [10] :

$$
\Sigma(q)=\sum_{\alpha, \beta=2}^{m} k_{\alpha} k_{\beta} \cdot S_{\alpha \beta}(q)+\frac{b_{1}}{v_{1}} \sum_{\alpha=1}^{m}\left\langle n_{\alpha}\right\rangle\left(k_{\alpha}+b_{\alpha}\right) \cdot k T \cdot \chi_{T} \quad q<q^{*} \quad k_{\alpha}=b_{\alpha}-b_{1} \frac{v_{\alpha}}{v_{1}}
$$

où $k_{\alpha}$ est la longueur de contraste par rapport au $\mathrm{DE} \alpha=1$ (généralement le solvant ou la matrice). Le second terme de cette équation est relié à la compressibilité du système pondéré par les contrastes des divers diffuseurs élémentaires. A $q<q^{*}$, il est indépendant de $q$ et comme la diffusion incohérente due aux états isotopiques et de spins des atomes (voir paragraphe 3.1), il sera considéré comme un bruit de fond plat qu'il faudra soustraire [7]. L'équation (11) est l'équation générale de la section efficace de diffusion cohérente en diffusion de neutrons aux petits angles. Le terme de compressibilité est souvent omis, car négligeable. C'est ce que nous supposerons dans la suite.

\subsection{Facteur de forme, facteur de structure}

Considérons un ensemble de $n$ objets identiques de $N$ diffuseurs élémentaires de longueur de contraste $k$ par rapport à un milieu (solvant). C'est un système binaire et la section efficace ne dépend que d'un seul facteur de structure, $\Sigma(q)=k^{2} S(q)$. Il est utile de séparer les contributions provenant des interférences entre centres diffuseurs appartenant à un même objet de ceux appartenant à des objets différents. L'expression de $S(q)$ comporte $n^{2} N^{2}$ termes, dont $n N^{2}$ proviennent des interférences intra-objets et $n(n-1) N^{2} \cong n^{2} N^{2}$ concernent les interférences inter-objets :

$$
\Sigma(q) / k^{2}=n \sum_{i, j}^{N} \exp \left(i q\left(r_{i}^{\alpha}-r_{j}^{\alpha}\right)\right)+n^{2} \sum_{i, j, \alpha \neq \beta}^{N} \exp \left(i q\left(r_{i}^{\alpha}-r_{j}^{\beta}\right)\right)
$$

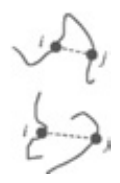


En introduisant les grandeurs normalisées :

On obtient

$$
\begin{aligned}
& P(q)=\frac{1}{N^{2}} \sum_{i, j}^{N} \exp \left(i q\left(r_{i}^{\alpha}-r_{j}^{\alpha}\right)\right) \\
& Q(q)=\frac{1}{N^{2}} \sum_{i, j, \alpha \neq \beta}^{N} \exp \left(i q\left(r_{i}^{\alpha}-r_{j}^{\alpha}\right)\right) \\
& \frac{\Sigma(q)}{k^{2}}=n \cdot N^{2} \cdot P(q)+n^{2} \cdot N^{2} \cdot Q(q)
\end{aligned}
$$

$P(q)$ est le facteur de forme de l'objet et $Q(q)$ le facteur de structure de l'ensemble. Ils rendent compte respectivement de la forme des objets et de leurs interactions.

Dans un système dilué, il n'y a pas de corrélations entre DE appartenant à des objets différents. L'intensité diffusée vaut $n N^{2} P(q)$, ce qui permet de déterminer directement le facteur de forme de l'objet. On remarque aussi que lorsque $q$ tend vers zéro, $P(q) \rightarrow 1$. La mesure de $\Sigma(q->0)$ donne le nombre total de diffuseurs dans la solution. Si on connaît la concentration totale de DE dans l'échantillon, on détermine le nombre de DE de l'objet (i.e. sa masse).

\subsection{Intensité diffusée aux petits angles}

Considérons d'abord un système dilué d'objets. L'intensité diffusée se réduit au facteur de forme $P(q)$. En utilisant l'hypothèse d'isotropie, on écrit l'équation (12) sous la forme [12] :

$$
P(q)=\frac{1}{N^{2}} \sum_{i, j}^{N, N}\left\langle\frac{\sin \left(q r_{i j}\right)}{q r_{i j}}\right\rangle
$$

Aux petites valeurs de $q$, cette équation est facilement développée :

$$
P(q)=\frac{1-q^{2} R_{g}^{2}}{3} \quad q R_{g}<<1
$$

où $R_{g}$ est le rayon de giration de l'objet, défini comme :

$$
R_{g}^{2}=\frac{1}{N} \sum_{i}\left\langle r_{i}^{2}\right\rangle \quad \sum_{i} r_{i}=0
$$

C'est une longueur quadratique qui caractérise la dimension globale de l'objet.

Notons que l'équation (14) est valable quelle que soit la forme de l'objet mais uniquement pour $q R_{g}<<1$, dans le domaine appelé domaine de Guinier. La détermination du rayon de giration d'un objet est donc possible sans en connaître la forme, simplement en approximant l'intensité diffusée à l'équation (14) mais dans le bon domaine de $q$ ! Lorsque les objets sont grands $\left(R_{g}>20 \mathrm{~nm}\right)$, il $s$ 'avère difficile d'atteindre un domaine de vecteurs de diffusion suffisamment petits pour que la condition $q R_{g}<<1$ soit respectée. Il faut alors ajuster l'intensité diffusée à des facteurs de forme particuliers (sphère, cylindre, bâton, ellipse, coquille...[13]). Selon les formes, la validité de l'équation (14) s'étend à des valeurs de $q$ au-delà de $q R_{g}<1$. Notons que lorsqu'on connaît l'expression du facteur de forme de l'objet, on peut aisément tenir compte d'une distribution de taille dans le calcul du facteur de forme.

Dans le cas d'objets orientés, il faut revenir à l'écriture vectorielle du vecteur de diffusion et développer le facteur de forme $P\left(q_{x}\right)$, pour toute direction $x$ :

$$
P\left(q_{x}\right) \cong 1-q_{x}^{2} R_{x}^{2} \quad q_{x} R_{x}<<1
$$

où $R_{x}$ est la distance moyenne d'inertie [14]. 
Là encore, on peut être amené à ajuster l'intensité diffusée à des facteurs de forme après les avoir moyennés par une distribution d'orientation particulière.

Lorsque les systèmes sont plus concentrés, les objets se rapprochent et interagissent. L'effet de ces interactions modifie l'intensité diffusée aux petits vecteurs de diffusion. On étudiera alors l'évolution des rayons de giration, de la position d'un pic de corrélation...avec la concentration. Ces évolutions donneront des éléments pour déterminer la forme des objets et leurs interactions. Par exemple, une variation en $c^{1 / 3}$ de la position d'un pic de corrélation indique une simple loi de dilution d'objets sphériques, alors qu'une variation en $c^{1 / 2}$ indique la présence d'objets « plan » (lamelles).

Bien souvent, on effectue des mesures à des concentrations d'objets de plus en plus faibles; ces mesures permettent l'extrapolation à concentration nulle de l'intensité diffusée afin d'obtenir le facteur de forme de l'objet isolé. Ce type de mesures est très fréquent en DNPA, surtout en physico-chimie ou il est souvent aisé de diluer les systèmes étudiés. Dans le cas général, les méthodes de variation des contrastes succinctement décrites au paragraphe 4 devront être mises en IJuvre pour déterminer expérimentalement à la fois la forme et les interactions des objets en solution.

\subsection{Intensité diffusée dans le domaine intermédiaire}

Aux plus grandes valeurs de $q, q \cdot R_{g} \geq 4$ et $q \cdot \lambda_{D E} \leq 1$, où $\lambda_{D E}$ est la taille du diffuseur élémentaire, le facteur de forme $P(q)$ varie en $q^{-\alpha}$, où $\alpha$ dépend de la forme de l'objet. Le domaine $\frac{4}{R_{g}} \leq q \leq \frac{1}{\lambda_{D E}}$ est appelé domaine intermédiaire. La mesure de $\alpha$ permet souvent d'identifier la forme de l'objet. La Figure 4 montre l'intensité diffusée dans le domaine intermédiaire par quelques objets non orientés.

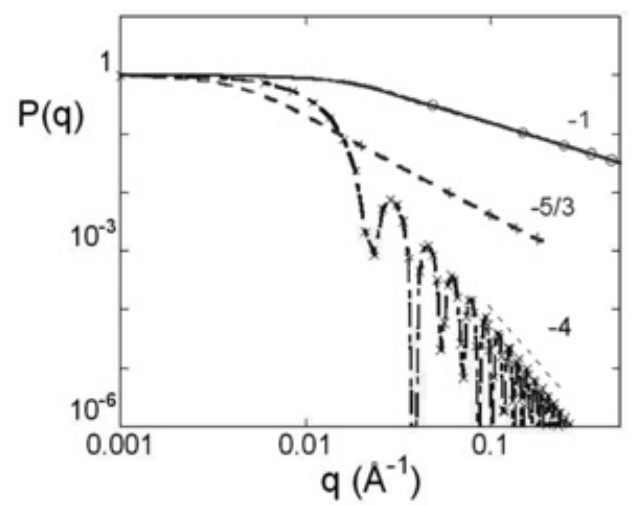

Figure 4. Comportements asymptotiques de facteurs de forme $(\times)$ d'une sphère $\left(\mathrm{R}=200 \AA ; R_{g}=\sqrt{\frac{3}{5}} R\right.$ $\left.P(q) \sim q^{-4}\right),(\bigcirc)$ d'un bâton $\left(\mathrm{L}=200 \AA ; R_{g}=\sqrt{\frac{L^{2}}{12}} P(q) \sim q^{-1}\right)$, (+) d'une chaîne de polymère en bon solvant $\left(R_{g}=200 \AA, \lambda=10 \AA ; P(q) \sim q^{-5 / 3}\right)$.

Quand il est inférieur à 3, l'exposant $\alpha$ est simplement relié à la dimension fractale des objets diffractants. Dans un espace à $d$ dimensions, la transformée de Fourier d'un objet isotrope de densité $g(r) \propto \frac{1}{r^{n}}$ vaut $S(q) \propto \frac{1}{q^{d-n}}$. Pour un objet fractal de dimension $D_{f}, g(r) \propto \frac{r^{D_{f}}}{r^{d}}$, et le facteur de forme varie selon la loi de puissance

$$
S(q) \propto \frac{1}{q^{d-d+D_{f}}}=\frac{1}{q^{D_{f}}}
$$


L'exposant $\alpha$ est égal à $D_{f}$. Le tableau ci-dessous illustre cette relation pour des objets de formes simples.

\begin{tabular}{|l|l|l|}
\hline & Dimension fractale & Variation de $S(q)$ \\
\hline Bâton & $D_{f}=1$ & $q^{-1}$ \\
\hline Disque & $D_{f}=2$ & $q^{-2}$ \\
\hline Polymère idéal (gaussien) & $D_{f}=\frac{1}{v} ; v=\frac{1}{2}$ & $q^{-\frac{1}{v}} ; q^{-2}$ \\
à volume exclu & $; v=\frac{3}{5}$ & $q^{-\frac{5}{3}}$ \\
\hline
\end{tabular}

Pour mettre en évidence ces formes différentes d'objets, on représente souvent le produit $q^{\alpha} P(q)$ qui devient constant aux grands angles. Le comportement gaussien d'une chaîne de polymère apparaît ainsi dans la représentation de Kratky $q^{2} P(q)$.

La Figure 5 montre une telle représentation pour des couches ultra-minces de polystyrène [15] de 10 et $100 \mathrm{~nm}$. Ces études de conformation de chaînes de polymères confinées dans des films d'épaisseur inférieure à leurs rayons de giration en volume ont très récemment fait l'objet de nombreuses expériences par diffusion de neutrons tant sur la structure [16,17,18], que sur la dynamique $[19,20]$ surtout depuis l'observation de l'abaissement d'environ $50 \mathrm{~K}$ de la température de transition vitreuse pour des films de 10 nm [21,22]. Les observations faites sont à ce jour encore l'objet de controverses. D'après la Figure 5, la conformation locale semble plus étendue dans le cas d'une chaîne confinée, le signal variant plutôt comme $q^{-1}$, que dans le volume ou la diffusion attendue suit une loi en $q^{2}$ (soit un plateau en $q^{2} I(q)$ ), caractéristique d'une conformation gaussienne.

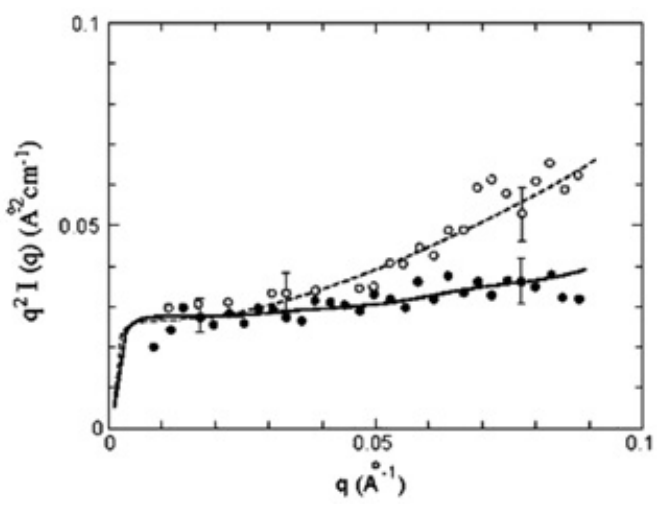

Figure 5. Représentation de Kratky $q^{2} I(q)$ de l'intensité diffusée par un fondu de polystyrène $(\mathrm{H}, \mathrm{D})$ de masse moléculaire 660000 confiné en couches minces d'épaisseur (•) 1000 Å (comportement gaussien en volume) et (o) $100 \AA$ (augmentation de la rigidité locale; $q^{-2}->q^{-1}$ ). Figures extraites de la référence [15].

En général, une fois la forme de l'objet identifiée, il faut effectuer des ajustements des intensités diffusées avec des modèles de facteurs de forme pour déterminer les paramètres de l'objet. De nombreux modèles ont été publiés dans la littérature ; la référence [13] en donne une bonne revue. Dans ces calculs, il est souvent tenu compte d'une distribution de tailles et parfois aussi de la résolution du spectromètre [23]. 


\subsection{Exemple : les polymères fondus}

La fonction de diffusion d'un fondu de $n$ polymères (chacun ayant $N$ monomères), schématisé sur la Figure 6, est selon (12) la somme de deux fonctions de diffusion, $P(q)$ et $Q(q)$, facteurs de structure intra et inter-chaînes.
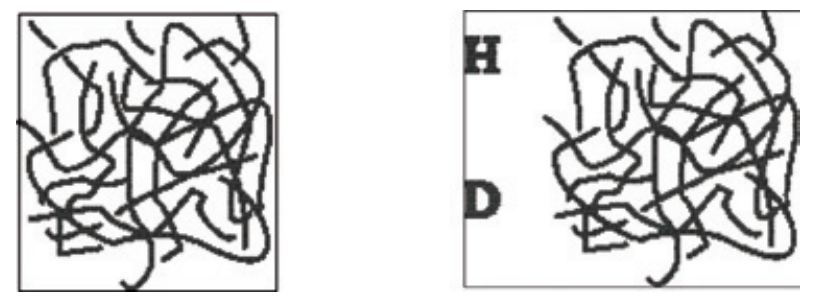

Figure 6. Représentations schématiques de fondus (a) de polymères identiques, (b) mélange de chaînes marquées et non marquées.

Lorsque les chaînes sont identiques, il n'y a pas de fluctuations de concentration et les fluctuations de densité sont faibles. Comme la plupart des liquides ordinaires, ce fondu est incompressible à grande échelle et l'intensité diffusée est très faible si on excepte l'éventuelle diffusion incohérente discutée au paragraphe 2 :

$$
S(q) \approx \frac{n N}{v} k T \chi_{T} \approx 0 q<q *
$$

Le facteur de forme du polymére ne pourra être obtenu qu'en créant artificiellement des fluctuations de concentration, c'est-à-dire en « marquant » certaines chaînes. La solution est d'utiliser des mélanges de chaînes hydrogénées et deutériées. Si les différentes chaînes ont le même degré de polymérisation $N$ et si les interactions entre paires de monoméres H-H, D-D et H-D sont les mêmes ${ }^{24}$, les facteurs $P(q)$ et $Q(q)$ des chaînes marquées et non marquées sont identiques. À cause de l'incompressibilité globale du systéme, l'intensité diffusée par unité de volume s'exprime uniquement en fonction du facteur de structure des chaînes D, par exemple :

$$
I(q)=\left(b_{H}-b_{D}\right)^{2} S_{D D}(q)
$$

où $b_{H}$ et $b_{D}$ sont les longueurs de diffusion des monoméres H et D. $S_{D D}(\mathrm{q})$ s'écrit :

$$
S_{D D}\left(c_{D}, q\right)=c_{D} P(q)+c_{D}^{2} Q(q)
$$

$C_{D}$ est la concentration de monoméres deutériés. L'intensité diffusée est nulle comme il se doit quand il n'y a pas de chaînes marquées $\left(c_{D}=0\right)$, elle doit l'être aussi quand il n'y a que des chaînes marquées $\left(c_{D} v=\varphi_{D}=1\right.$ où $v$ est le volume d'un monomére et $\varphi_{D}$ la fraction volumique de monoméres deutériés) : $S_{D D}\left(c_{D} v, q\right)=0$. Dans un fondu, on a donc la relation $Q(q)=-v P(Q)$. Par conséquent,

$$
I(q)=\left(b_{H}-b_{D}\right)^{2} \varphi(1-\varphi) P(q) / v
$$

Quelle soit la concentration en chaîne marquée, l'intensité diffusée ne mesure que le facteur de forme d'une seule chaîne au milieu des autres. Ce résultat très important pour étudier les fondus de polyméres est résumé dans le théoréme 50/50 [25]. En effet, d'aprés l'équation (16), l'intensité diffusée est maximum pour une fraction en volume de $50 \%$ de chaînes marquées. La DNPA associée à la technique de marquage est la seule technique qui permette de déterminer le facteur de forme de polyméres à l'état fondu.

La Figure 7 montre la diffusion de fondus de polystyrène. La DNPA a permis de mesurer aux petits $q$ le rayon de giration des chaînes, ainsi que la variation $R_{g} \propto M^{1 / 2}$ caractéristique du comportement 
gaussien de chaînes linéaires. Aux grands vecteurs de diffusion, la dépendance en $q^{-2}$ de l'intensité diffusée confirme le caractére gaussien des polyméres à l'état fondu.

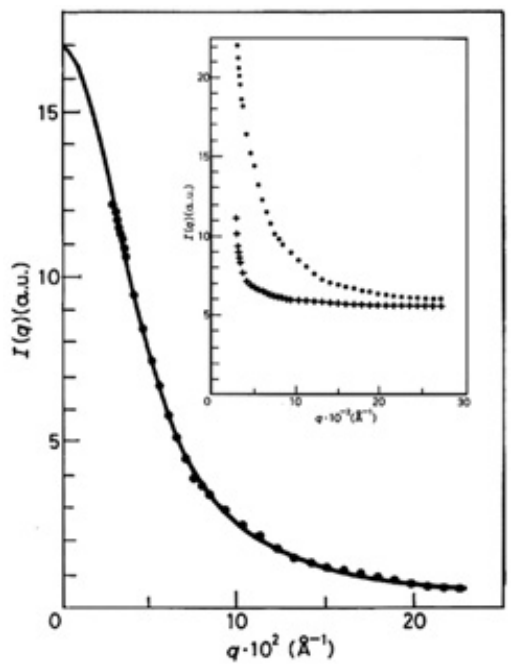

Figure 7. Intensité cohérente diffusée par un fondu de chaînes de polystyrène $\mathrm{H}$ et $\mathrm{D}$ de masse moléculaire 21000. Aux petits vecteurs de diffusion, on mesure le rayon de giration des chaînes, $R_{g}=38 \AA$. En insert, le signal diffusé brut par un fondu de chaînes identiques hydrogénées : le signal est quasiment plat (incohérent) sur toute la gamme de $q$; lorsqu'on marque certaines chaînes, un fort signal diffusé est alors mesuré. Figures extraites de la référence [25]. Aux grands vecteurs de diffusion, on observe les fluctuations locales des chaînes ; l'intensité diffusée varie en $q^{-2}$ et est indépendante de la masse moléculaire.

\section{MÉTHODES DE VARIATION DE CONTRASTE EN DNPA}

Varier les termes de contraste qui sont les coefficients des facteurs de structures partiels dans l'expression de l'intensité diffusée (équation (12)) permet de séparer les différentes contributions et de déterminer ces facteurs de structures. En pratique, la variation des contrastes est faite en remplaçant une partie des constituants des objets étudiés par des isotopes de longueurs de diffusion différentes.

Dans ce paragraphe, nous décrirons les techniques les plus usuelles comme l'annulation de contraste (paragraphe 4.1.) qui est très utile dans les études de systémes ternaires, ainsi que des techniques plus élaborées comme la méthode de contraste moyen nulle qui permet de déterminer le facteur de forme d'objets dans une solution où les interactions ne sont pas négligeables (paragraphe 4.2.). D'autres subtilités du contraste en DNPA sont résumées dans la revue de J.P. Cotton [26].

\subsection{Variation de contraste. Annulation de contraste}

Les systèmes étudiés sont souvent des systémes complexes, multi-composants. Si l'on désire étudier la distribution spatiale d'une espèce bien définie au sein d'un objet, il est nécessaire de le marquer afin que sa contribution dans la diffusion se distingue du reste.

La diffusion d'un mélange ternaire, deux espèces (2) et (3) et un solvant (1), s'écrit d'après (11) sous la forme :

$$
I(q)=k_{2}^{2} S_{2}(q)+2 k_{2} k_{3} S_{23}(q)+k_{3}^{2} S_{3}(q) \text { avec }\left(k_{\alpha}\right)^{2}=\left(b_{\alpha}-b_{1} \frac{v_{\alpha}}{v_{1}}\right)
$$


Les $k_{\alpha}$ sont les longueurs de contraste déjà introduites et les $S_{\alpha \beta}$ les facteurs de structures partiels des différentes espèces.

Le principe de la variation de contraste repose sur le remplacement d'un noyau par l'un des isotopes. $99 \%$ des cas concernent le couple H/D. Cette substitution n'engendre pas de perturbation chimique significative sauf dans les cas où les effets des liaisons hydrogène sont importants. Lorsque que l'on mélange deux solvants isotopiques, la longueur de diffusion moyenne du mélange devient :

$$
b_{1}(x)=x b_{1 D}+(1-x) b_{1 H}
$$

Comme $x$ varie entre 0 et 1 , il est possible de faire varier $b_{1}$ sur une large gamme de valeurs. C'est la technique de variation de contraste [27]. En effectuant trois mesures avec des proportions différentes de solvant deutérié, il est possible de déterminer les trois fonctions $S_{i j}(q)$.

De plus, il est souvent possible de choisir la proportion de solvant marqué telle que

$$
k_{2}=\left(b_{2}-b_{1}(x) \frac{v_{2}}{v_{1}}\right)=0
$$

on ne mesure alors que $I(q)=k_{3}^{2} S_{3}(q)$ (voir Figure $8(\mathrm{~b})$ ). De même, en réalisant $k_{3}=0$, on mesure $I(q)=k_{2}^{2} S_{2}(q)$ (voir Figure $8(\mathrm{~b})$ ). C'est la condition d'annulation du contraste ou de « contrast matching » en franglais.

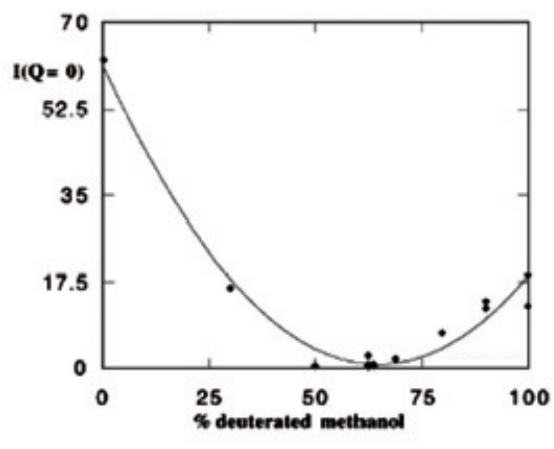

(a)

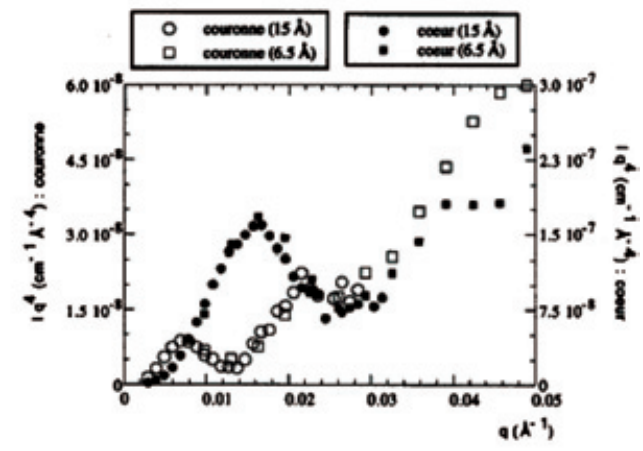

(b)

Figure 8. (a) Intensité diffusée extrapolée à angle nul dans une expérience de variation de contraste sur un verre poreux, le Vycor. (b) Intensité diffusée par des particules colloïdales de silice couvertes de polymères greffés. Les symboles pleins correspondent à un contraste ou l'on n'observe que l'intensité diffusée par le cIJur de silice, les symboles vides correspondent au contraste nul avec la silice, on n'observe que l'intensité diffusée par la couronne de polymère. La représentation choisie $q^{4} I(q)$ fonction de $q$ met en évidence les différences de comportement asymptotique.

Cette méthode est utilisée avec les solvants organiques courants ou avec l'eau. La détermination de $\mathrm{x}$ est faite par calcul à partir des longueurs de diffusion et des volumes molaires ou expérimentalement si on peut disperser séparément les espèces dans le solvant et déterminer la fraction qui annule l'intensité diffusée du mélange binaire solvant-espèce (voir Figure 8(a)).

\subsection{Contraste Moyen Nul}

\section{Application aux systémes binaires en interaction}

Un des intérêts du marquage isotopique est de pouvoir observer la structure d'un seul objet au milieu des autres, bien que tous les objets interagissent entre eux et que l'intensité diffusée dépende des interactions. 
La méthode générale de séparation entre facteur de forme, ne dépendant que de la géométrie des objets diffusants individuels, et facteur de structure, dépendant des interactions, est de réaliser plusieurs solutions en variant la fraction d'objets deutériés tout en conservant constant le nombre total des objets. On résout le système d'équations (20) ci-dessous ou on extrapole les courbes obtenues à concentration en objets deutériés nulle.

Une seconde méthode consiste à utiliser un mélange de solvant normal et de solvant deutérié, tel que la densité de longueur de diffusion moyenne du mélange soit égale à la moyenne de celles de l'objet hydrogéné et de l'objet deutérié. Cette deuxième méthode est appelée méthode de contraste moyen nul ou « zero average contrast $(\mathbf{Z A C}) »$. Par rapport à la méthode précédente, elle a l'avantage de ne requérir qu'une seule mesure.

Considérons une solution de $n$ objets semblables dont une fraction $\phi$ est marquée et $(1-\phi)$ non marquée. Les facteurs de structure partiels (équation (17)) s'écrivent sous la forme suivante :

$$
\begin{aligned}
& S_{D D}(q)=n \phi P(q, n)+n^{2} \phi^{2} Q(q, n) \\
& S_{H D}(q)=n^{2} \phi(1-\phi) Q(q, n) \\
& S_{H H}(q)=n(1-\phi) P(q, n)+n^{2}(1-\phi)^{2} Q(q, n)
\end{aligned}
$$

Nous avons séparé le facteur de forme $P(q, n)$ du facteur de structure $Q(q, n)$. L'intensité diffusée totale s'écrit alors :

$$
\begin{gathered}
I(q)=n\left(\phi k_{D}^{2}+(1-\phi) k_{H}^{2}\right) P(q, n)+n^{2}\left(\phi k_{D}+(1-\phi) k_{H}\right)^{2} Q(q, n) \\
\operatorname{avec}\left(k_{\alpha}\right)^{2}=\left(b_{\alpha}-b_{1} \frac{v_{\alpha}}{v_{1}}\right)^{2}
\end{gathered}
$$

On voit sur cette équation l'intérèt de faire une expérience de diffusion avec un mélange de solvants de longueur de diffusion moyenne $b_{1}(x)=x b_{1 D}+(1-x) b_{1 H}$ telle que

$$
\phi k_{D}+(1-\phi) k_{H}=0
$$

On annule alors la contribution du terme d'interaction et l'intensité diffusée ne dépend que du facteur de forme $P(q, n)$.

La principale difficulté consiste à disposer d'objets marqués et non marqués identiques et d'effectuer les mesures dans un mélange de solvants où l'intensité est réduite à cause d'un contraste inférieur dans le mélange que dans le solvant pur. Il faut aussi déterminer le meilleur bruit de fond [7,8] à soustraire au signal de la solution à contraste moyen nul.

\section{Application aux systèmes ternaires et aux systèmes confinés}

La diffusion par un système à trois composants ( 2 objets et un solvant ou une matrice) contient trois facteurs de structure partiels (cf. équation (17)) avec autant de fonctions de diffusion inter- et intra- objets. C'est le cas d'une solution de « liquidecomplexe» (polymères, surfactants ou colloïdes) confinée dans un milieux poreux ou d'une suspension de particules couvertes de polymères greffés. La détermination des formes et des interactions requiert de nombreuses expériences où l'on variera ou annulera le contraste des objets.

Cependant, on ne s'intéresse souvent qu'à un seul des objets, souvent celui qui est confiné. On peut alors obtenir le facteur de forme de l'objet étudié en une seule mesure. Il faut pour cela

- annuler le contraste du milieu confinant, équation (19), pour ne mesurer que la fonction de diffusion de l'objet confiné,

- vérifier de plus la condition de contraste moyen nul, équation (21), pour l'objet confiné afin de ne mesurer que son facteur de forme. 
La première condition fixe la proportion $x$ de solvant marqué qui éteint le milieu confinant. Elle fixe aussi la fraction $\phi$ d'objets marqués confinés. Cette méthode est illustrée dans les exemples de systèmes confinés détaillés paragraphe 6 . Notez qu'outre la difficulté de disposer d'objets marqués et non marqués identiques, la double condition d'annulation de contraste et de contraste moyen nul conduit à une valeur de contraste de l'objet confiné assez faible.

\section{DNPA SUR DES INTERFACES}

La DNPA permet d'accéder à la structure d'interfaces entre objets de petite taille, par exemple entre des sphères d'une espèce dispersée dans un solvant ou une matrice, et l'espèce hôte. Si l'interface est parfaitement lisse à l'échelle $q^{-1}$, on observe une variation de l'intensité diffusée en loi de Porod [28] en $q^{-4}$, simplement proportionnelle à la quantité de surfaces diffusantes par unité de volume (surface spécifique, $S / V)$. Si l'interface est plus diffuse, comme pour une interpénétration des deux milieux, ce signal en $q^{-4}$ peut être multiplié par la transformée de Fourier du profil de l'interface. L'analyse du signal diffusé permettra de déterminer le profil de l'interface.

\subsection{Variations autour de la loi de Porod}

Un milieu homogène ne diffuse pas. Seules des variations spatiales ou des fluctuations de densité contribuent à l'intensité diffusée à un angle non nul. Dans de nombreux matériaux divisés (suspensions colloïdales, milieux poreux, phases de surfactant) ces variations de densité sont localisées aux interfaces et dans un certain sens, la diffusion est un processus purement interfacial.

En général l'intensité diffusée dépend explicitement de la géométrie des interfaces et on utilise la diffusion pour obtenir des informations sur cette géométrie. Il existe cependant un régime dit asymptotique ou "de Porod" où la diffusion ne dépend que de la structure locale des interfaces et non de la géométrie globale du milieu. Nous allons montrer que la loi de Porod est caractéristique des interfaces planes mais peut être généralisée aux interfaces rugueuses et au cas des profils de concentration interfaciaux.

\section{Loi de Porod. Surfaces planes}

Quand on observe un milieu à deux phases pour un vecteur de diffusion $q$ grand devant la courbure de la surface de séparation des milieux, celle-ci apparaît plane à l'échelle spatiale $q^{-1}$. Les contributions de portions planes d'interfaces de taille $q^{-1}$ sont additives et l'intensité diffusée devient proportionnelle à l'aire totale $S$ de la surface interne du milieu. C'est le régime de Porod.

On démontre souvent la loi de Porod dans l'espace direct, il est cependant intéressant et instructif de l'établir directement dans l'espace réciproque. La méthode est due à de Gennes. On applique directement la formule de base à une interface unique séparant deux milieux. On calcule d'abord l'amplitude de diffusion, puis l'intensité et on moyenne ensuite sur toutes les orientations de la surface. Les milieux sont notés 1 et 2 , leurs densités de longueur de diffusion sont $n_{1}$ et $n_{2}$. Les coordonnées d'un point $\mathbf{r}$ sont $z$ perpendiculairement à l'interface et $\rho=(x, y)$ parallèlement. L'orientation du vecteur de diffusion $\boldsymbol{q}$ par rapport à l'interface est arbitraire, ses composantes sont :

$$
q_{B}=\left(q_{x}, q_{y}\right)=(q \sin \theta \cos \varphi, q \sin \theta \sin \varphi) \quad \text { et } \quad q_{z}=q \cos \theta
$$

L'amplitude de diffusion est la transformée de Fourier de la densité de longueur de diffusion $n(\mathbf{r})$ :

$$
a(q)=\int d^{3} r n(r) e^{i q r}=\int d^{2} \rho\left(\int_{-\infty}^{0} d z n_{1} e^{i q r}+\int_{0}^{\infty} d z n_{2} e^{i q r}\right)
$$


En négligeant les contributions rapidement oscillantes provenant des bornes à l'infini, on obtient :

$$
a(q)=\frac{\left(n_{1}-n_{2}\right)}{i q_{z}} \int d^{2}\left(\rho e^{i q_{/ /} \rho}\right)
$$
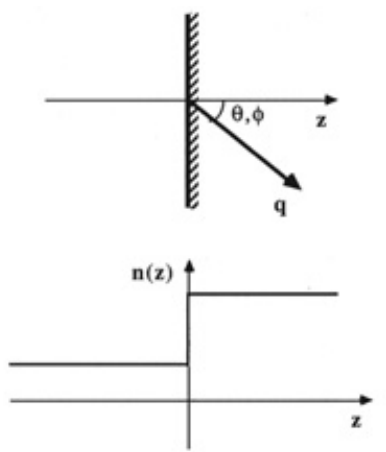

Figure 9. (a) En haut. Géométrie de la diffusion par une interface unique. (b) En bas, profil de densité de longueur de diffusion en marche d'escalier conduisant à la loi de Porod.

Le facteur $\left(n_{1}-n_{2}\right) / i q_{z}$ est la transformée de Fourier de l'échelon de densité à travers l'interface.

L'intensité diffusée $i(\mathbf{q})=\frac{1}{V}|a(\mathbf{q})|^{2}$ implique le calcul de l'intégrale singulière

$$
\int d^{2} \rho d^{2} \rho^{\ni} e^{i q_{/ /}\left(\rho-\rho^{3}\right)}=(2 \pi h)^{2} S \delta\left(q_{x}\right) \delta\left(q_{y}\right)=4 \pi q^{-2} \delta(\sin \theta \cos \varphi) \delta(\sin \theta \sin \varphi)
$$

$S$ est l'aire totale de la surface.

Cette expression n'est non nulle que pour $\theta=0$ et $\theta=\pi$. -Le vecteur de diffusion est alors en géométrie de réflexion, c'est à dire exactement perpendiculaire à l'interface.

Dans un milieu poreux ou une suspension colloïdale, les interfaces ne sont pas orientées dans une direction définie, nous devons donc calculer la moyenne sur toutes les orientations de la surface par rapport à q. L'intensité diffusée rapportée à l'unité de volume est donc :

$$
i(q)=\frac{1}{4 \pi} \int d \varphi \sin \theta d \theta i(\mathbf{q})
$$

Après intégration, on arrive à la loi de Porod exprimée sous la forme :

$$
i(q)=2 \pi\left(n_{1}-n_{2}\right)^{2} \frac{S}{V} \frac{1}{q^{2}} \frac{1}{q^{2}}
$$

$S / V$ est l'aire interfaciale par unité de volume.

Un facteur $1 / q^{2}$ provient du carré de la transformée de Fourier du profil échelon de densité, l'autre facteur $1 / q^{2}$ provient de la moyenne angulaire sur le terme de réflexion. Ainsi la loi de Porod ne résulte que des contributions des réflexions du faisceau incident sur les portions d'interface de l'échantillon qui ont l'orientation convenable pour fournir un faisceau réfléchi vers un angle de diffusion donné. En ce sens, la loi de Porod n'est rien d'autre que la loi de réflectivité de Fresnel calculée dans l'approximation de Born. 


\section{Surfaces rugueuses}

Si une surface est rugueuse, son aire dépend de l'échelle d'observation. Une loi de diffusion analogue à celle de Porod existe pour ce cas et exprime exactement la constatation précédente. La présentation que nous proposons est inspirée d'une démonstration non publiée de T. Witten.

Nous considérons un objet dense, homogène et uniforme dans son volume mais dont la surface est rugueuse quoiqu'en moyenne plate à grande échelle. La dimensionnalité de l'objet et de l'espace dans lequel il est plongé est $d$. L'intensité diffusée est comme d'habitude

$$
I(q) \infty \sum_{i, j}\left\langle\exp \left(-i \vec{q} \cdot\left(\vec{r}_{i}-\vec{r}_{j}\right)\right)\right\rangle
$$

Pour calculer $I(q)$ dans le domaine $q^{-1}<R$, où $R$ est la taille de l'objet, nous pavons l'objet par des "boules de cohérence" de rayon $q^{-1}$. Les boules qui sont entièrement dans le volume de l'objet ne contribuent qu'à la diffusion vers l'avant, car la répartition de densité y est uniforme. Pour évaluer l'intensité diffusée, il suffit de considérer la contribution des boules ne recouvrant que la surface. Nous notons $N_{S}\left(q^{-1}\right)$ le nombre total de boules pavant la surface et $g_{S}\left(q^{-1}\right)$ le nombre d'éléments diffusants (DE) à l'intérieur d'une boule. L'amplitude diffusée totale par les monomères à l'intérieur d'une boule est proportionnelle à $g_{S}\left(q^{-1}\right)$ et l'intensité correspondante est proportionnelle à $g_{S}\left(q^{-1}\right)$. Comme les intensités diffusées par les boules s'ajoutent de façon incohérente (sans interférence), l'intensité totale est simplement l'intensité diffusée par une boule multipliée par le nombre total de boules, soit

$$
I(q) \sim N_{S}\left(q^{-1}\right) \cdot g_{S}^{2}\left(q^{-1}\right)
$$

Évaluons d'abord $g_{S}$. Il faut remarquer pour cela que les éléments diffusants occupent toujours une fraction finie du volume d'ordre $q^{-d}$ d'une boule, par conséquent $g_{S}$ est proportionnel au rapport :

$$
g_{S} \propto\left(\frac{q^{-1}}{a}\right)^{d}
$$

où $a$ est une taille moléculaire.

$N_{S}$ le nombre de boules de cohérence sur la surface dépend de la rugosité. Ce n'est pas en général une fonction simple de la variable $q^{-1}$. Cependant nous pouvons le relier à $\sum\left(q^{-1}\right)$, l'aire apparente mesurée à la résolution $q^{-1}$. En effet cette aire est proportionnelle à l'aire totale des boules :

$$
\sum\left(q^{-1}\right) \propto N_{S}\left(q^{-1}\right)\left(q^{-1}\right)^{d-1}
$$

Finalement l'intensité diffusée s'exprime selon la relation :

$$
I(q) \sim N_{S}\left(q^{-1}\right) \cdot g_{S}^{2}\left(q^{-1}\right) \propto \frac{1}{a^{2 d}} \frac{\sum\left(q^{-1}\right)}{q^{d+1}}
$$

Pour $d=3$

$$
I(q) \propto \frac{1}{a^{6}} \frac{\sum\left(q^{-1}\right)}{q^{4}}
$$

Ceci n'est rien d'autre que la loi de Porod, mais l'aire $\sum\left(q^{-1}\right)$ dépend du vecteur de diffusion, c'est à dire de l'échelle d'observation.

Si la surface est auto-similaire ou fractale, le nombre de boules de rayon $r$ qui recouvrent la surface est une loi de puissance :

$$
N_{S}(r) \propto\left(\frac{R}{r}\right)^{D_{S}}
$$


$R$ est la taille maximale de l'objet, $D_{S}$ est la dimension fractale de la surface $\left(d-1<D_{S}<d\right)$. Toutes les autres quantités varient alors en loi de puissance :

$$
\begin{aligned}
\sum(r) & \propto R^{D_{S}} r^{(d-1)-D_{S}} \\
I(q) & \propto \frac{R^{D_{S}}}{a^{2 d}} \frac{1}{q^{2 d-D_{S}}}
\end{aligned}
$$

Pour $d=3$,

$$
I(q) \propto \frac{R^{D_{S}}}{a^{6}} \frac{1}{q^{6-D_{S}}}
$$

L'exposant de décroissance de l'intensité diffusée est alors compris entre 3 et 4 . La loi précédente, découverte par Bale et Schmitt, explique certaines observations sur la lignite, les charbons et de nombreuses roches poreuses.

\section{Diffusion par des structures interfaciales}

De nombreux matériaux divisés sont susceptibles de posséder des structures interfaciales épaisses et diffuses, caractérisées par un profil de concentration perpendiculaire à la surface : double couche électrostatique d'ions au voisinage d'une surface chargée, couches de polymères adsorbés ou greffés en présence de solvant ou d'autres polymères, interfaces créées par séparation de phase etc...Il est intéressant La diffusion centrale des neutrons est bien adaptée à l'étude des structures interfaciales s'étendant en volume et la réflectométrie à celle des surfaces macroscopiquement planes. Nous prendrons comme exemple particulier le cas de couches polymériques, qui a été beaucoup étudié théoriquement et expérimentalement.

\section{a) Intensité dans le régime de Porod}

Un des intérêts de la démonstration précédente de la loi de Porod est qu'elle est facilement généralisable au cas des structures interfaciales planes, caractérisées par un certain profil de concentration $\phi(z)$, qui sera pour nous la fraction volumique en polymère à la distance $z$ d'une paroi.

Remarquons qu'en général l'intensité diffusée ne dépend pas seulement d'un profil de concentration moyen dans la couche mais aussi des corrélations de concentration à l'intérieur de la couche. En effet, dans une expérience de diffusion aux petits angles où la surface étudiée a une orientation quelconque, le vecteur de diffusion a en général une composante perpendiculaire à la surface et une autre composante parallèle à celle-ci. On sonde à la fois la structure de la couche perpendiculairement à la surface et parallèlement à elle. Dans le premier cas, on est sensible au profil de concentration, dans le second aux corrélations parallèles. L'intensité diffusée totale sera donc la somme d'un terme $\bar{I}$ de profil et d'un terme de corrélations $\widetilde{I}$.

Nous supposons comme précédemment le substrat plan et nous calculons l'amplitude de diffusion comme la transformée de Fourier de la densité de longueur de diffusion :

$$
a(\mathbf{q})=\int d^{2} \rho e^{i \mathbf{q} / / \rho}\left[\frac{\left(n_{g}-n_{s}\right)}{i q_{z}}+\left(n_{p}-n_{s}\right) \int_{0}^{\infty} d z \phi(z) e^{i q_{z} z}\right]
$$

$n_{g}, n_{s}$ et $n_{p}$ sont respectivement les densités de longueurs de diffusion du solide ( $g$ signifie "grain"), du solvant et du polymère.

L'intensité correspondante est $I(\mathbf{q})=\frac{1}{V}\left[a(\mathbf{q})^{2}\right]$. En introduisant la fluctuation locale $\delta \phi(\mathbf{r})=\phi(\mathbf{r})-$ $\langle\phi(\mathbf{r})\rangle$ et en moyennant sur les orientations, on isole immédiatement le terme de corrélation :

$$
\begin{aligned}
\widetilde{I}(q) & =\left(n_{p}-n_{s}\right)^{2 \widetilde{S}_{p p}(q)} \\
\widetilde{S}_{p p}(q) & =\frac{S}{V} \int d z_{1} d z_{2} d y_{1} d y_{2}\left\langle\delta \phi\left(0,0, z_{1}\right) \delta \phi\left(x_{2}, y_{2}, z_{2}\right)\right\rangle \frac{\sin q r_{12}}{q r_{12}}
\end{aligned}
$$


$r_{12}$ est la distance entre les deux points $\left(0,0, z_{1}\right)$ et $\left(x_{2}, y_{2}, z_{2}\right)$. L'expression précédente est entièrement analogue à l'expression de l'intensité diffusée en volume.

Le terme de profil $\bar{I}(q)$ ne dépend que de la transformée de Fourier $\alpha(q)$ du profil total moyen de densité de longueur de diffusion :

$$
\begin{aligned}
\bar{I}(q) & =2 \pi \frac{S}{V} \frac{1}{q^{2}}|\alpha(q)|^{2} \\
\alpha(q) & =\frac{\left(n_{g}-n_{s}\right)}{i q}+\left(n_{p}-n_{s}\right) \int_{0}^{\infty} d z \phi(z) e^{i q z}
\end{aligned}
$$

\section{b) Facteurs de structure interfaciaux}

Nous déduisons de l'expression de l'intensité totale les expressions des différents facteurs de structure partiels que l'on sépare par des expériences de variations des contrastes. Il y a trois facteurs de structure partiels reliés aux différentes corrélations solide-solide, solide-polymère et polymère-polymère et

$$
I(q)=\left(n_{g}-n_{s}\right)^{2} S_{g g}(q)+\left(n_{p}-n_{s}\right)^{2} S_{p p}(q)+2\left(n_{p}-n_{s}\right)\left(n_{g}-n_{s}\right) S_{p g}(q)
$$

Le facteur de structure solide-solide est simplement le facteur de structure du solide nu dans le régime asymptotique. Il est donné par la loi de Porod usuelle. Les deux facteurs de structure du polymère sont donnés par les expressions suivantes :

$$
\begin{aligned}
& S_{p p}(q)=2 \pi \frac{S}{V} q^{-2}\left|\int_{0}^{\infty} d z \phi(z) e^{i q z}\right|^{2}+\widetilde{S}_{p p}(q) \\
& S_{p g}(q)=-2 \pi \frac{S}{V} q^{-3} \int_{0}^{\infty} d z \phi(z) \sin q z
\end{aligned}
$$

Ces équations appellent plusieurs remarques :

i) le facteur de structure polymère - polymère est le plus simple à mesurer, il suffit d'annuler le contraste entre le solide et le solvant, c'est à dire d'imposer $n_{g}=n_{s}$.

ii) le facteur de structure croisé polymère - solide $S_{p g}(q)$ est le plus simple d'un point de vue théorique car il ne dépend que du profil de concentration moyen et pas des corrélations interfaciales. Ceci résulte de l'interférence "hétérodyne" entre l'amplitude diffusée par le solide supposé homogène et l'amplitude diffusée par la couche.

iii) on peut en principe utiliser les informations obtenues sur le profil à partir de $S_{p g}$ pour séparer dans $S_{p p}$ la contribution des corrélations $\widetilde{S}_{p p}$.

c) Limites aux petits et grands angles

Comme pour la diffusion en volume, il est intéressant d'examiner les comportements généraux de l'intensité diffusée dans les deux limites où l'inverse du vecteur de diffusion est beaucoup plus grand ou plus petit que l'épaisseur caractéristique des couches $h$.

i) Dans le domaine des petits vecteurs de diffusion, $q$ h plus petit que 1 (mais $q$ encore dans le domaine de Porod), la diffusion ne dépend que des caractéristiques globales de la couche interfaciale :

- la quantité totale de matière accrochée à la surface $\gamma=\int_{0}^{\infty} d z \phi(z)$,

- l'épaisseur ou plus précisément les premiers moments du profil de concentration $\left\langle z^{n}\right\rangle=\gamma^{-1} \int_{0}^{\infty} d z z^{n} \phi(z)$. 
En développant l'exponentielle des expressions précédentes en série, on obtient les analogues surfaciques de la loi de Guinier en volume :

$$
\begin{aligned}
& q^{2} S_{p p}(q)=2 \pi \frac{S}{V} \gamma^{2}\left(1-q^{2}\left(<z^{2}>-<z>^{2}\right)\right)+q^{2} \widetilde{S}_{p p}(0)+o\left(q^{4}\right) \\
& q^{2} S_{p g}(q)=2 \pi \frac{S}{V} \gamma<z>\left(1-\frac{1}{6} q^{2} \frac{<z^{3}>}{<z>}\right)+o\left(q^{4}\right)
\end{aligned}
$$

ii) Aux très grand angles $(q h>>1)$, L'intensité diffusée est dominée par la contribution de la discontinuité de densité à la paroi, si le profil de concentration n'est as singulier. En intégrant l'expression de l'amplitude diffusée par partie, on obtient un comportement asymptotique strictement analogue à la loi de Porod.

$$
\begin{aligned}
& S_{p p}(q)=2 \pi \frac{S}{V} \phi_{S}^{2} \frac{1}{q^{4}}+\widetilde{S}_{p p}(q) \\
& S_{p g}(q)=2 \pi \frac{S}{V} \phi_{S} \frac{1}{q^{4}}
\end{aligned}
$$

$\phi_{S}$ est la fraction volumique en polymère directement en contact avec la surface.

Dans cette limite particulière, la combinaison des équations précédentes permet de séparer simplement $\widetilde{S}_{p p}(q)$.

\subsection{Exemple d'une couche de polymère adsorbée}

Un exemple de structure interfaciale est celui d'une couche de polymères adsorbée sur une surface. Quoique les quantités adsorbées soient souvent assez faibles (typiquement $1 \mathrm{mg} / \mathrm{m}^{2}$ ), il est quand même possible d'observer des couches de polymères par diffusion de neutrons. La première expérience a été faite sur des chaînes de PEO (poly(éthylène-oxyde)) adsorbées sur des latex de polystryrène deutérié en condition de contraste nul dans de l'eau lourde [29]. Négligeant volontairement la contribution du terme de corrélation $\widetilde{S}_{p p}(q)$, ces auteurs ont interprété les données expérimentales avec une contribution de profil moyen. Comme ce profil n'existe que pour l'espace $z>0$, il existe certaines relations entre les parties réelle et imaginaire de sa transformée de Fourier (analogues aux relations de KramersKronig pour la susceptibilité diélectrique en optique), et l'on peut inverser en principe l'intensité diffusée mesurée pour en déduire le profil de concentration. Le profil de concentration ainsi obtenu décroît très rapidement. On observe une très grande différence entre l'épaisseur déduite par diffusion de neutrons et les épaisseurs hydrodynamiques mesurées par d'autres techniques. Ces différences indiquent qu'une petite fraction des monomères appartient à de grandes boucles ou à des extrémités qui dominent le comportement hydrodynamique. Ces boucles ne sont pas faciles à observer par diffusion de neutrons car le signal est dominé par la partie dense de la couche adsorbée tout près de la surface. Ceci explique la difficulté des études expérimentales de couches adsorbées.

La structure de ces couches est souvent analysée à l'aide de théorie de champ moyen [30]. La description la plus élégante et la plus concise est donnée par de Gennes [31] en terme de lois d'échelle. L'idée est qu'en bon solvant, les chaînes adsorbées se comportent localement comme dans une solution semi-diluée en volume et construisent un réseau transitoire dont la maille est localement proportionnelle à la distance de la paroi (voir Figure 10). La structure est donc auto-similaire. La théorie des polymères en solution et l'expérience montre que la taille $\xi$ (ou la longueur de corrélation) de la maille d'une solution semi-diluée dépend de la fraction volumique en monomères selon la loi $\xi=a \phi^{-3 / 4}$ (où a est une longueur moléculaire de d'ordre de la taille d'un monomère). L'hypothèse de proportionnalité $\xi(z) \propto z$ conduit à un profil de concentration singulier variant en

$$
\phi(z)=\left(\frac{a}{z}\right)^{4 / 3}
$$


La partie non divergente de la transformée de Fourier de ce profil est une loi de puissance en $q^{1 / 3}$. On attend alors que le facteur de structure croisé polymère-solide varie en $q^{-8 / 3}$ [32] :

$$
S_{p g}(q)=2 \pi \frac{S}{V}(2 a)^{4}(q a)^{-8 / 3}
$$

Cette loi de puissance a été vérifiée expérimentalement par variation de contraste en DNPA sur des chaînes de PDMS de masse 270000 adsorbées sur de la silice poreuse en présence cyclohexane[33]. Elle a été confirmée par des expériences de réflectivité de neutrons sur des chaînes de PDMS adsorbées à la surface libre d'une solution de toluène [34].

La Figure 10 montre les données obtenues dans un domaine de vecteurs de diffusion plus large que celui de la référence [33] sur du PDMS de plus grand masse $(M=470000)$ en solution dans du dichlorométhane. La pente de la courbe du facteur de structure croisé, $S_{p g}$ vaut -2.7 , en très bon accord avec l'exposant théorique $-\frac{8}{3}$. Les courbes obtenues pour l'autre facteur de structure, polymèrepolymère $S_{p p}$, confirment cet accord [35].

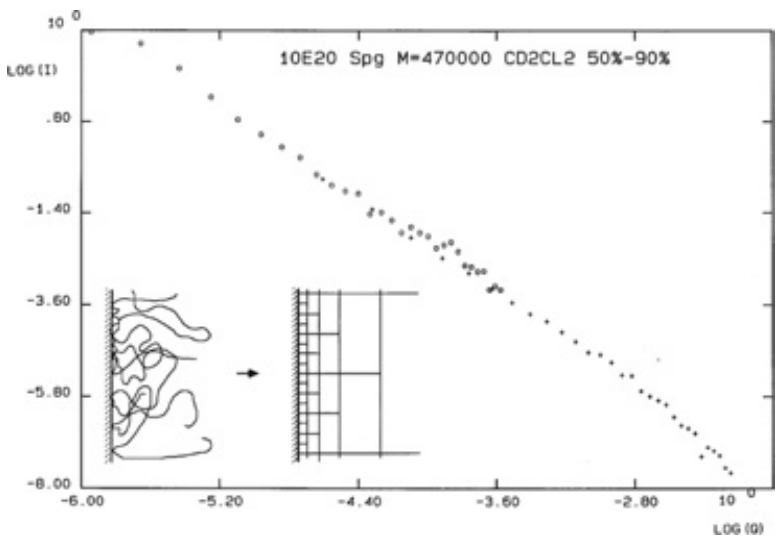

Figure 10. Variation du facteur de structure croisé polymère-grain pour du PDMS de masse 470000 dans du dichlorométhane et adsorbé sur de la silice poreuse (coordonnées logarithmiques). En insert, le schéma de la variation auto-similaire de la structure à la surface.

\subsection{Exemple de profil de concentration de polymère greffé}

Les couches de polymères greffés sur une surface sont faites de différentes manières : par adsorption forte d'un groupe terminal, qui peut être un bloc de copolymère, ou par réaction chimique de groupes fonctionnels terminaux avec des sites réactifs de la surface. En bon solvant, les monomères se repoussent par interaction de volume exclu. Si la densité de greffage est suffisamment grande pour que les chaînes greffées se recouvrent, cet effet conduit à un allongement des chaînes perpendiculairement à la surface qui est limité par une force de rappel d'origine entropique [36].

Les résultats les plus spectaculaires obtenus par DNPA dans les études de couches de polymères ont été obtenus avec des couches de polymères greffés [37,38,39]. La raison est qu'on peut greffer de grandes quantités de polymères sur une surface. En pratique, la quantité attachée à la surface atteint des valeurs de l'ordre de $30 \mathrm{mg} / \mathrm{m}^{2}$, environ 30 fois plus grande que celle obtenue en adsorbant des polymères en solution diluée. Le signal de diffusion est très grand. Il est alors relativement simple de déterminer les différents facteurs de structure, quantité de polymères greffés, profil de concentration par les techniques d'annulation ou de variation des contrastes. Il est aussi possible de varier la qualité de solvant et d'étudier le gonflement des couches [37] (Figure 11). Dans ces études, la difficulté majeure est la synthèse de couches de polymères greffés de grande masse moléculaire bien contrôlées. 
Un des résultats obtenus est en mauvais solvant que les couches sont collapsées. Les facteurs de structures sont alors décrit par un profil de concentration en créneau. La fraction volumique $\phi(z)$ est constante sur une épaisseur $h$. La transformée de Fourier de ce profil particulièrement simple est $\phi(q)=$ $\frac{\phi_{S}}{i q}\left(e^{i q h}-1\right)$ où $\phi_{S}$ est la fraction de polymère située juste à la surface du solide.

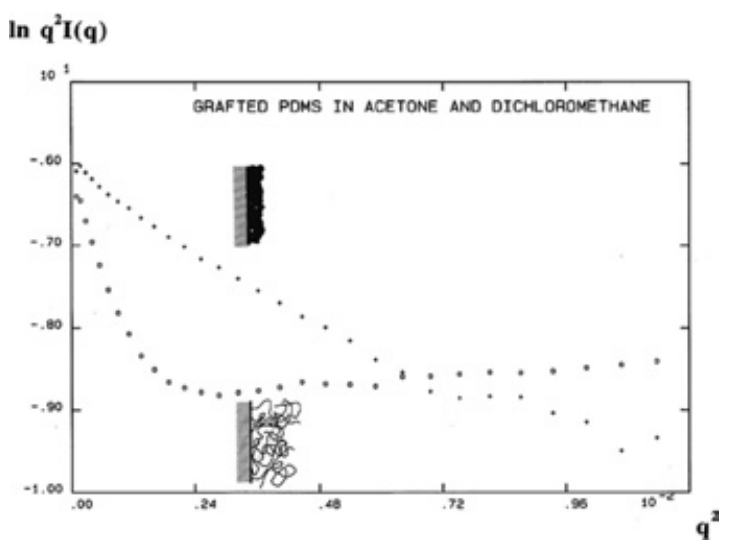

Figure 11. Couche de PDMS $\left.(M=57000), \Gamma=3.8 \mathrm{mg} / \mathrm{m}^{2}\right)$ greffé sur la silice poreuse en bon et en mauvais solvant (respectivement dichlorométhane et acétone) observé à contraste nul entre la silice et le solvant.

Les facteurs de structure correspondant s'expriment :

$$
\begin{aligned}
q^{4} S_{p p}(q) & =2 \pi \frac{S}{V}\left(2 \phi_{S}^{2}\right)(1-\cos q h) \\
q^{4} S_{p g}(q) & =2 \pi \frac{S}{V} \phi_{S}(1-\cos q h)
\end{aligned}
$$

Aux petits angles, les développements de ces deux équations permettent d'obtenir l'épaisseur de la couche collapsée et la quantité totale de polymère, $\phi_{S} h[35,37]$.

L'analyse complète des spectres de diffusion et des facteurs de structures partiels permet également de remonter aux profils de concentration. Un exemple est donné sur la Figure 12 pour le cas d'une couche greffée en bon solvant [39]. Le profil théorique attendu est parabolique. L'accord entre la théorie et l'expérience est excellent.

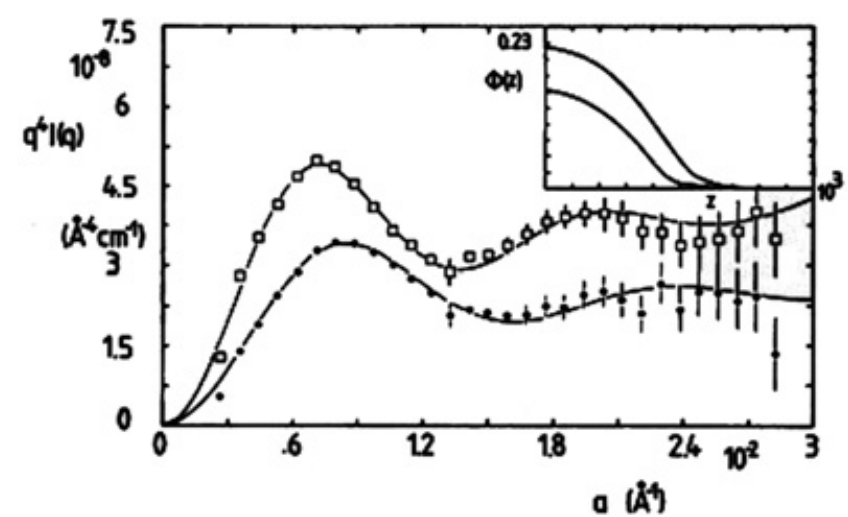

Figure 12. Facteur de structure croisé polymère-solide de couches de polystyrène greffé sur de la silice poreuse en bon solvant (dichlorométhane) pour des densités de greffage différentes. En inséré, les profils de concentration qui décrivent au mieux les données [39]. 


\section{DIFFUSION DES NEUTRONS PAR DES POLYMÈRES CONFINÉS}

L'étude de polymères dans des milieux de dimensionnalité restreinte est importante d'un point de vue théorique. Elle concerne aussi un certain nombre de situations pratiques : la chromatographie d'exclusion stérique, la récupération assistée du pétrole ou encore la fabrication de matériaux composites obtenus par exemple en intercalant des polymères dans des structures lamellaires d'argile. Le cas d'un confinement bidimensionnel entre plaques ou lamelles est différent de celui d'un confinement uni-dimensionnel dans des canaux.

\subsection{Polymères confinés}

Les rares expériences de diffusion des neutrons effectués sur ces systèmes ont porté pour le moment sur le cas de polymères confinés dans des milieux poreux comme le verre Vycor dont les pores sont localement cylindriques $[40,41,42]$. Un bon moyen d'étudier le confinement bidimensionnel de polymères est d'effectuer des expériences de réflectivité ou de diffraction des neutrons sur des films ultra-minces déposés sur des substrats plans $[15,16,18,17]$.

Le premier problème à résoudre est de confiner les polymères. Une chaîne unique ne rentre pas spontanément dans un pore de largeur $D$ inférieure à son rayon de giration car la réduction d'entropie imposée par le confinement est trop importante. Il faut donc utiliser une force extérieure pour confiner les chaînes. Pour des chaînes neutres en bon solvant, le plus simple est d'appliquer une pression osmotique. Si la solution est « semi-diluée », cette pression est principalement due aux effets de volume exclu et la répulsion entre les chaînes force le confinement si la concentration est suffisamment grande. P.G. de Gennes et M. Daoud [43] ont montré que des chaînes de polymères neutres rentrent dans des fentes ou des canaux dès que la longueur de corrélation $\xi$ de la solution semi-diluée est inférieure à $D$ (Figure 13). Pour des pores de Vycor, dont le rayon est de $3.5 \mathrm{~nm}$, cela correspond à des concentrations supérieures à $20 \%$ (en poids/volume). Cette prédiction a été vérifiée expérimentalement. La pénétration dans du verre Vycor de chaînes de polystyrène dont la masse est comprise entre 35000 et 800000 Da est presque totale dès que leur concentration en solution dépasse effectivement $20 \%$.

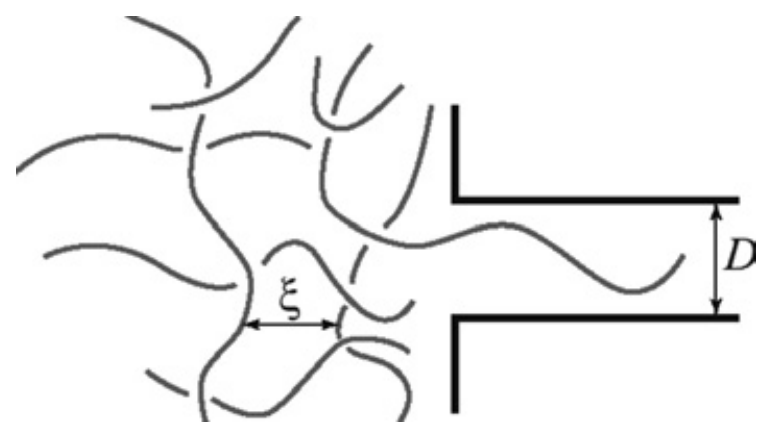

Figure 13. Les chaînes d'une solution semi-diluée de polymères ne rentrent dans des pores de diamètre $D$, que si la longueur de corrélation $\xi$ est inférieure à $D$.

L'observation de la structure des chaînes se fait par diffusion de neutrons. Cette technique est ici absolument indispensable. Il est en effet nécessaire d'annuler la contribution du milieu poreux à la diffusion qui domine en pratique le signal mesuré.

La première condition est d'annuler le contraste entre le solvant utilisé et le milieu poreux mais ce n'est pas suffisant. Dans les cas étudiés jusqu'à présent la concentration en polymère dans le pore est relativement élevée (de l'ordre de 20\%) et les chaînes confinées sont soit enchevêtrées, soit au contact l'une de l'autre. Comme dans les solutions en volume, il est difficile d'obtenir des informations sur la 
structure d'une chaîne au milieu des autres. À contraste nul entre le poreux et le solvant, le système apparaît à grande échelle comme un réseau de pores décoré par une solution homogène de polymère et l'intensité mesurée dans ces conditions renseigne principalement sur la structure du milieu poreux (Figure 14).

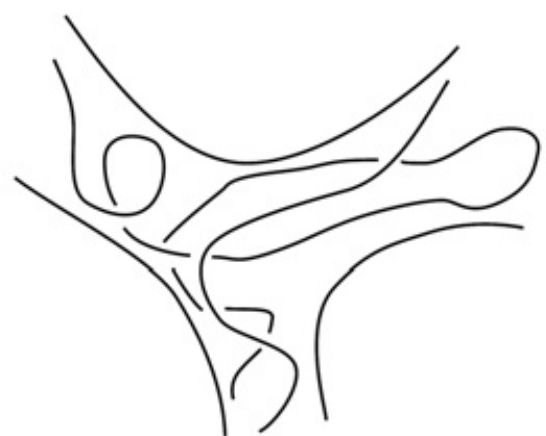

(a)

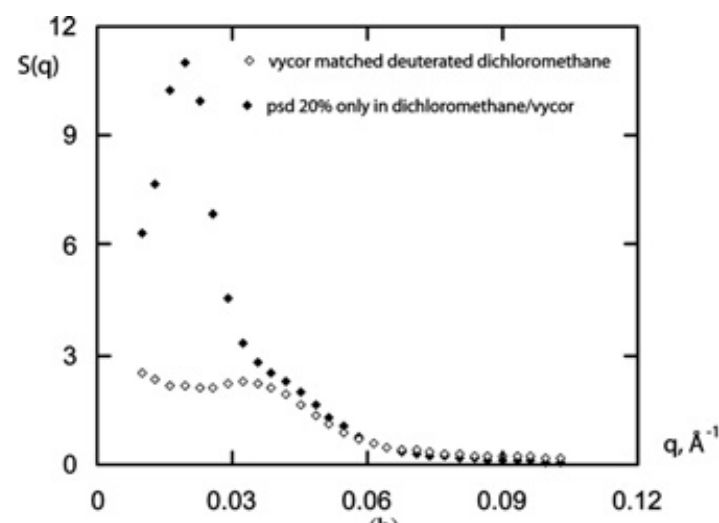

(b)

Figure 14. À gauche, schéma de chaînes confinées non marquées. À droite, en symbole creux, intensité des neutrons diffusés par le Vycor en présence de dichlorométhane deutérié au voisinage du point de contraste nul entre le milieux poreux et le solvant. En symbole plein, le même échantillon avec $20 \%$ de polystyrène deutérié confiné $(M=351000 \mathrm{Da})$. La forme du signal est identique à celle qu'on mesurerait sur du Vycor nu à plein contraste.

Pour observer l'effet du confinement sur les chaînes, il est nécessaire d'utiliser la méthode de contraste moyen nul exposée précédemment. Il faut à la fois annuler le contraste en le solvant et le milieu poreux et mélanger des chaînes de polymères hydrogénées et deutériées en proportion telle que la longueur de diffusion moyenne du polymère soit égale à celle du solvant. On mesure ainsi directement le facteur de structure d'une chaîne au milieu des autres (Figure 15).

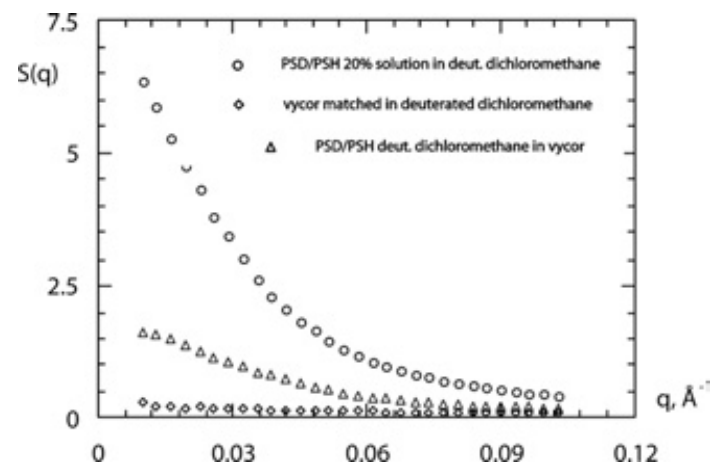

Figure 15. Diffusion par des polymères confinés (polystyrène, $\mathrm{Mw}=32$ 700) dans des conditions de contraste moyen nul. Comparaison avec des chaînes libres en solution dans les mêmes conditions de contrastes.

Le rayon de giration des chaînes confinées est toujours inférieur à celui des chaînes libres dans la solution équivalente. Ces mesures sont en accord avec les prédictions théoriques [43] établies pour des chaînes confinées dans un pore cylindrique quand les chaînes sont enchevêtrées et comprimées transversalement mais restent idéales à grande échelle dans la direction du cylindre à cause de l'écrantage du volume exclu (régime dit "de cigares semi-dilués"). 

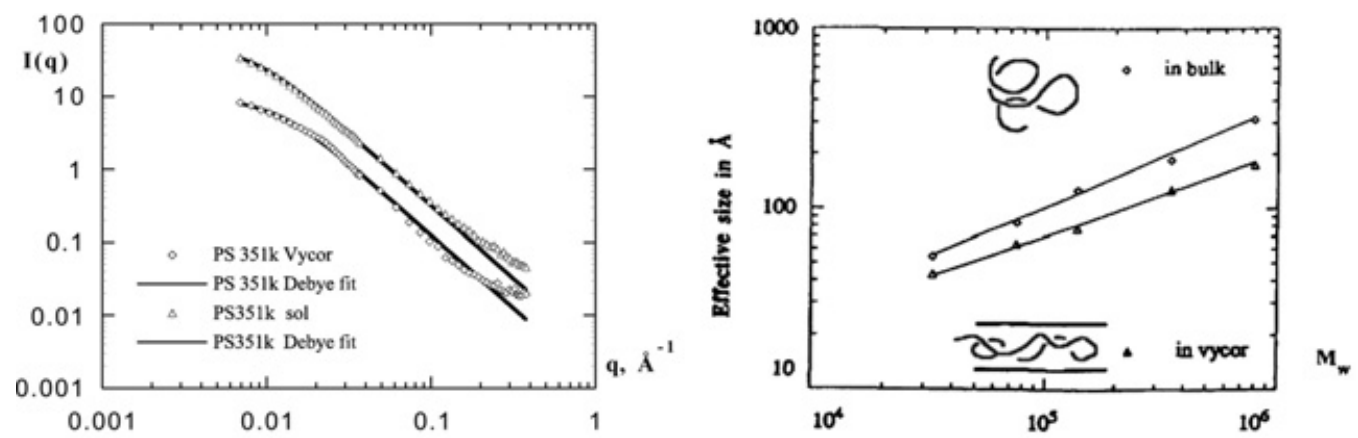

Figure 16. À gauche, comparaison entre les facteurs de forme de chaînes de polystyrène libres et confinées mesurés par la méthode de contraste moyen nul $(c=20 \%)$. À gauche, variation des rayons de giration en fonction de la masse pour des chaînes de polystyrène libres et confinées en solution semi-diluée $(c=20 \%)$.

\subsection{Polyélectrolytes confinés}

Plus récemment, la même technique a été appliquée à l'étude d'un polyélectrolyte confiné, le polystyrènesulfonante de sodium $[41,44]$, polymère chargé dont la conformation a été beaucoup étudiée en volume. La pénétration du polymère et sa structure dans les pores est alors dominée par des effets électrostatiques, notamment de confinement des contre-ions. Il est là aussi nécessaire que la solution soit suffisamment concentrée pour que les chaînes rentrent dans les pores. Le critère théorique d'entrée est que la longueur de Debye de la solution soit inférieure au diamètre des pores $D$. S'il existe quelques travaux théoriques $[43,45]$ décrivant la conformation de chaînes neutres confinées, il n'en n'est pas de même pour les polymères chargés. Malgré cela, les corrélations entre monomères chargés commencent à peine à être comprises en volume $[46,47,48,49]$.

Dans ce cas encore, il faut séparer les différentes contributions inter et intra chaînes, les corrélations entre le polymère et le milieu confinant... La mesure de l'intensité diffusée pour différents contrastes, en particulier le contraste moyen nul, permet de déterminer le facteur de forme d'une chaîne $P(q)$ et le facteur de structure intermoléculaire $Q(q)$.

Dans l'exemple étudié [41,44], le milieu confinant est le Vycor, présentant une porosité de $28 \%$ en volume et un diamètre moyen de pores de $7 \mathrm{~nm}$. Cette taille est inférieure aux rayons de girations de la plupart des chaînes chargées qui ont été étudiées. La diffusion de ce verre poreux seul présente un pic autour de $0.2 \mathrm{~nm}^{-1}$ provenant de corrélations ente pores distants de $30 \mathrm{~nm}$. Le mélange $\mathrm{D}_{2} \mathrm{O} / \mathrm{H}_{2} \mathrm{O}$ qui possède la même densité de longueur de diffusion que ce Vycor, et donc annule son contraste, correspond très précisément à $62.4 \%$ de $\mathrm{D}_{2} \mathrm{O}$. La condition du contraste moyen nul pour le polystyrène sulfonate dans le mélange qui annule le contraste du Vycor correspond exactement à 23:6 mol\% de polystyrène sulfonate deutérié. La Figure 17 montre les facteurs de forme $P(q)$ obtenus pour plusieurs masses moléculaires de polyélectrolytes confinés et non confinés.

D'après ses courbes (Figure 17), on déduit des valeurs de longueurs de persistance apparentes des chaînes de polystyrène sulfonate plus grandes qu'en solution libre. Le pic dans le facteur de structure est celui de la structure spinodale du Vycor : les chaînes très étirées révèlent les corrélations et la structure du milieu poreux à grande échelle. Le confinement se fait aussi sentir sur les corrélations entre chaînes. On observe et on interprète théoriquement que le facteur de structure inter-chaîne $Q(q)$ décroît aux grands angles en $\frac{1}{q^{2}}$ pour des chaînes libres et en $\frac{1}{q}$ pour les chaînes confinées. 

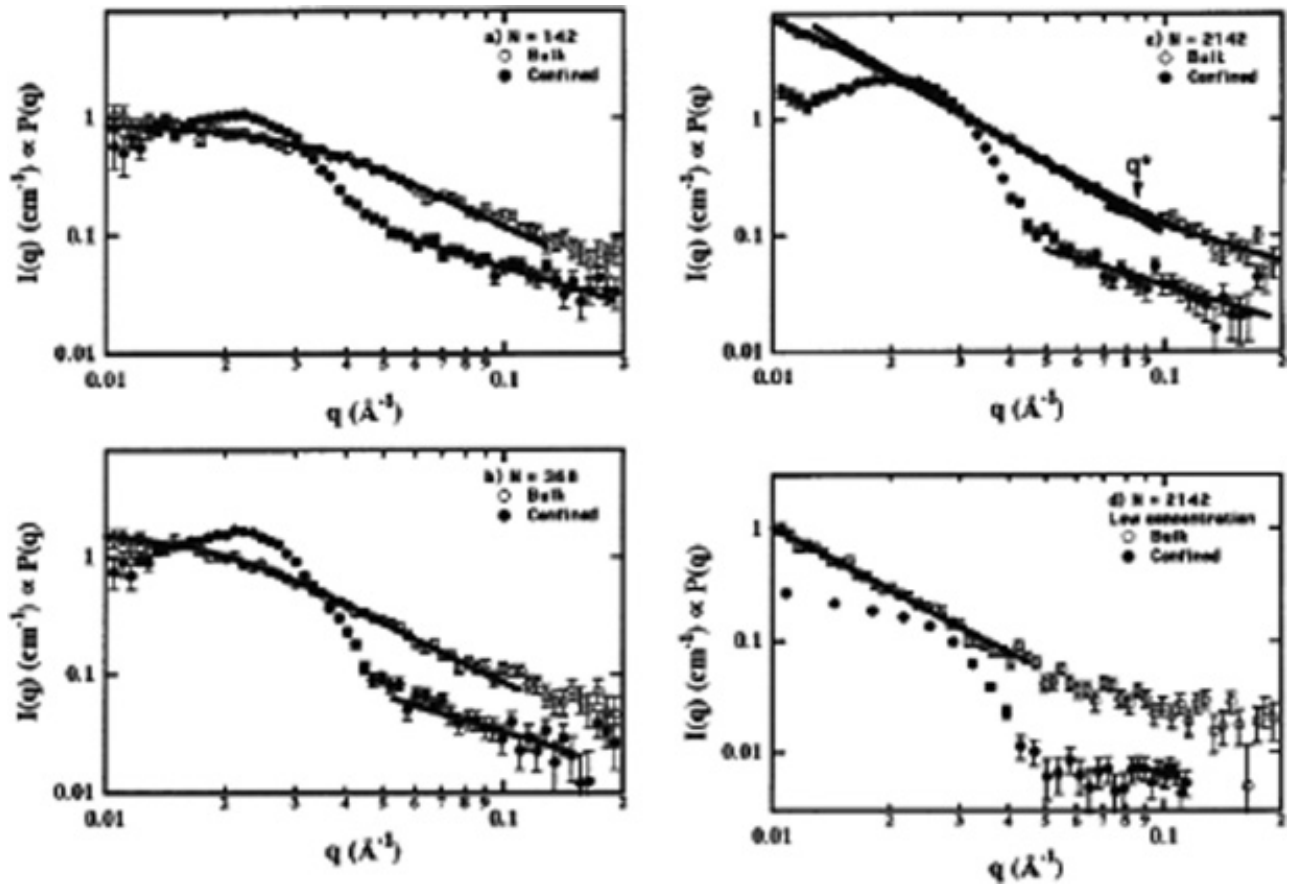

Figure 17. Facteurs de forme $P(q)$ de polyélectrolytes en condition de contraste moyen nul en volume (o) ou en géométrie confinée (•) : (a) de petite (b) de moyenne et (c) grande masse moléculaire à la concentration de $0.306 \mathrm{~g} \mathrm{~cm}^{-3}$ (d) de grande masse moléculaire à la concentration plus faible de $0.087 \mathrm{~g} \mathrm{~cm}^{-3}$. Les données obtenues en volume ont été ajustées au modèle de Sharp et Bloomfield de chaîne à longueur de persistance. Sur (c), on observe le changement de régime $q^{-2}$ et $q^{-1}$. Courbes issues de la référence 41 .

La DNPA permet aussi de préciser la structure du milieu confinant (diamètre et géométrie des pores). Par des expériences de variation, on peut discerner les pores ouverts ou non. En effet, dans ce dernier cas, il sera impossible d'annuler le signal diffusé, les mélanges de solvant ne pouvant pas entrer dans les pores fermés.

\section{CONCLUSIONS}

L'étude des surfaces et des systèmes confinés par diffusion des neutrons aux petits angles est l'un des domaines les plus prometteurs et les moins étudiés de la discipline. La technique de diffusion complète très utilement les études par réflectométrie pour l'étude des surfaces et elle se révèle indispensable pour l'étude des matériaux nanostructurés, micro-fabriqués ou réalisés par assemblages, qui se développent beaucoup actuellement. Elle est également très utile pour comprendre les mécanismes microscopiques à l'œuvre dans les applications de ces matériaux, en particulier au renforcement mécanique, à la filtration, à la séparation où à la conduction ionique. Les études statiques sont également le prélude indispensable aux études dynamiques dans lesquelles les notions et les techniques de contrastes présentées ici s'appliquent.

\section{Références}

[1] « Diffusion de Neutrons aux Petits Angles », J. Phys. IV France 9 (1999).

[2] « Neutrons et Magnétisme », J. Phys. IV France 11 (2001). 
[3] (a) Glattli dans, H., [2], p.19-31. (b) Heinemann, A., et Wiedenmann, A., J. Appl. Cryst. 36, 845 (2003).

[4] Sears, G. D., Neutrons News 3 (1992) 26.

[5] Cotton dans, J. P., [1], p.1-21.

[6] Fermon dans, C., [2], p.1-15.

[7] Cotton dans, J. P., "Neutrons, X-Ray and Light Scattering”, Lindner, P., Zemb, Th., Ed., North Holland (1991) p 19.

[8] Brûlet, A., Lairez, D., Lapp, A., Cotton, J. P., J. Appl. Cryst 40, (2007).

[9] Egelstaff dans, P., "An introduction to the liquid state" Academic Press N.Y. (1971).

[10] Cotton dans, J. P., "Neutrons, X-Ray and Light Scattering", Lindner, P., Zemb, Th., Ed., North Holland (1991) p 7.

[11] des Cloizeaux, J., Jannink, G., «Les Polymères en Solution ». Ed. de Physique (1987).

[12] Guinier, A., Fournet, G., "Small Angle Scattering of X-Rays", Wiley Interscience, New-York (1955).

[13] Pedersen dans, J. S. "Neutrons, X-Ray and Light Scattering”, Lindner, P., Zemb, Th., Ed., North Holland (2002) p 391-420.

[14] Pour une particule non orientée, $R x=R y=R z=R_{g} / \sqrt{3}$.

[15] Brûlet, A., Boué, F., Menelle, A., Cotton, J. P., Macromolecules 33, 997 (2000).

[16] Jones et al., Nature 400 (1999) 146 ; Macromolecules 34 (2001) 559.

[17] Muller Buschbaum et al., Eur. Phys. Lett 40 (2000) 210.

[18] Shuto et al, Macromolecules 26 (1993) 6589.

[19] (a) Frick, B. B., Dalnoki-Veress, K., Forrest, J. A., Dutcher, J., Murray, C., Higgins, A., Eur. Phys. J. E 12 (2003) S97. (b) Eur. Phys. J. E 12, s01 (2003).

[20] Soles, C. L., et al. Phys. Rev. Lett 88 (2002) 37401 ; Macromolecules 36 (2003) 373-379.

[21] Jones et al., Phys. Rev. E 63, 21501 (2001).

[22] Forrest et al., Phys. Rev Lett. 77, 10 (1996) 2002.

[23] (a) Pedersen, J. S., J. Phys. IV, C8 3 (1993) 491. (b) Lairez dans, D., [1], p.67-81.

[24] Certains polymères $\mathrm{H}$ et $\mathrm{D}$ ont un paramètre d'interaction $\chi$ qui tend à les séparer ; cet effet augmente avec la masse moléculaire.

[25] Cotton, J. P., Decker, D., Benoît, M., Farnoux, B., Higgins, J., Jannink, G., Ober, R., Picot, C., Des Cloizeaux, J., Macromolecules 7, 863 (1974).

[26] Cotton, J. P., Adv. Colloid Interface Sci. 69 (1996) 1-29.

[27] Stuhrmann, H. B., J. Appl. Cryst. 7, 173 (1974).

[28] Porod, G., Koll, Z., 124, 82 (1951).

[29] Cosgrove, T., Crowley, T. L., Vincent dans B., “Adsorption from solutions”, Ottewill, R., Ed., Academic Press (1983).

[30] Cohen Stuart, M., Cosgrove, T., Vincent, B., Adv. Coll. Interf. Sci. 24, 143 (1986).

[31] de Gennes, P. G., Ad. Coll. Interf. Sci. 27, 189 (1987). Macromolecules, 14, 1637 (1981) ; 15, 492 (1982).

[32] Auvray, L., de Gennes, P. G., Europhys. Lett, 2, 647 (1986).

[33] Auvray, L., Cotton, J. P., Macromolecules 20, 202 (1987).

[34] Guiselin, O., Lee, L. T., Farnoux, B., Lapp, A., J. Chem. Phys. 95, 6, 4632 (1991).

[35] Auvray, L., Auroy, P., in «Neutron, X-Ray and Light Scattering » Elsevier 1991.

[36] Alexander, S., J. Phys. (Paris) 38, 983 (1977).

[37] Auroy, P., Auvray, L., Léger, L., Macromolecules 24 (1991) 2523-2528.

[38] Auroy, P., Auvray, L., Léger, L., Phys. Rev. Lett. 66 (1991) 719-722.

[39] Auroy, P., Mir, Y., Auvray, L., Phys. Rev. Lett. 69, 93 (1992).

[40] Lal, J., Sinha, S. K., Auvray, L., J. Phys. II 7, 1597 (1997). 
[41] Gilbert, E. P., Auvray, L., Lal, J., Macromolecules 34 (2001) 4942-4998.

[42] Nieh, M.-P., Kumar Derek, S. K., Ho, L., Briber, R. M., Macromolecules 35 (2002) 6384-6391.

[43] Daoud, M., de Gennes, P. G., J. Phys. 38, 85 (1977).

[44] Lal, J., Gilbert, E. P., Auvray, L., Physica A 304 (2002) 244-248.

[45] Brochard, F., de Gennes, P. G., J. Phys. 40 (1979) L-399.

[46] Nierlich, M., Williams, C. E., Boué, F., Cotton, J. P., Daoud, M., Jannink, G., Picot, C., Moan, M., Wolf, C., Rinaudo, M., de Gennes, P. G., J. de Physique 40 (1979) 701.

[47] Boué, F., Cotton, J. P., Lapp, A., Jannink, G., J. Chem. Phys. 101 (3) (1994) 2562.

[48] Spiteri, M. N., Boué, F., Lapp, A., Cotton, J. P., Phys. Rev. Lett. 77 (26) (1996) 5218.

[49] Essafi, W., Lafuma, F., Willia, C. E. Eur. Phys. J. B 9 (2) (1999) 261. 